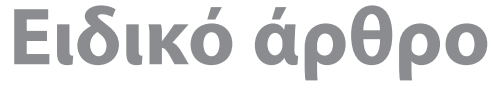 Special article
}

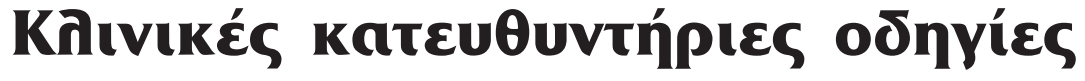

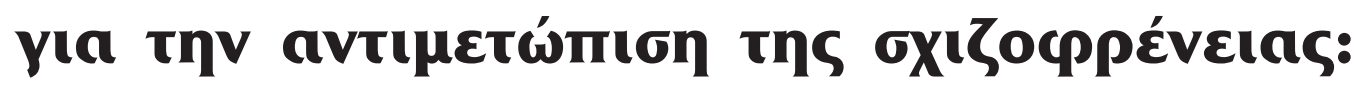

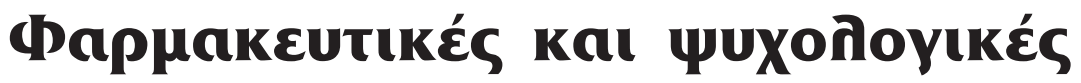

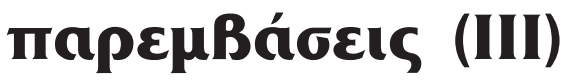

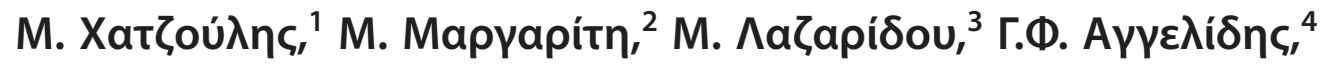

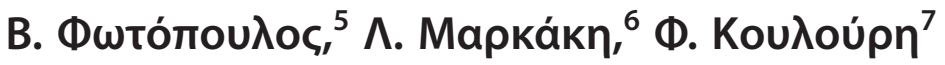

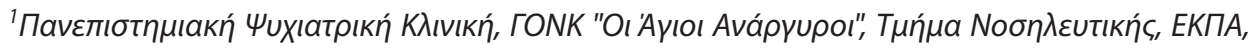

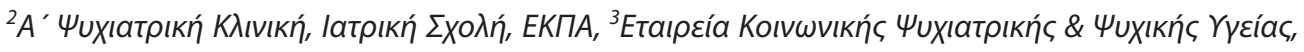

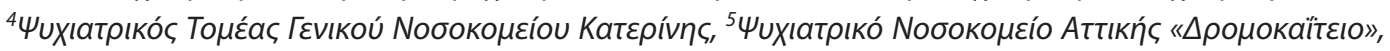

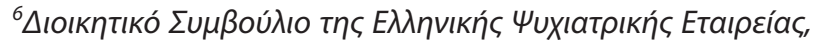

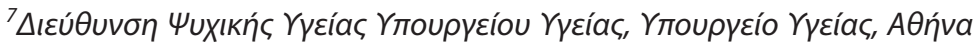

Uuxıatpıkń 2018, 29:303-315

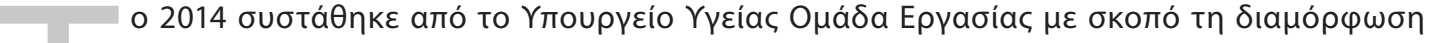

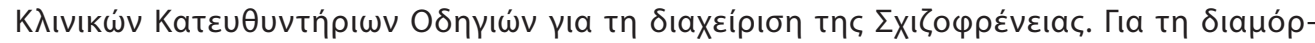

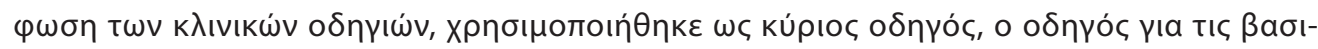

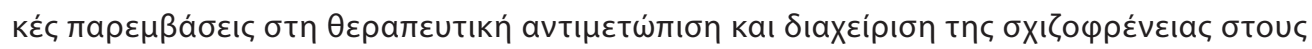

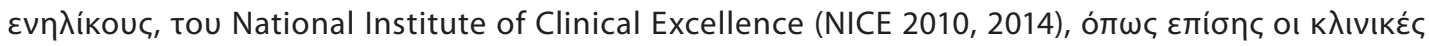

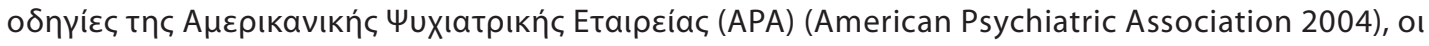

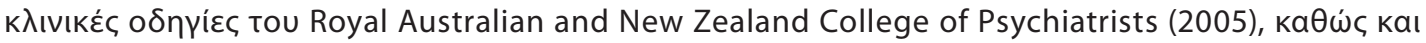

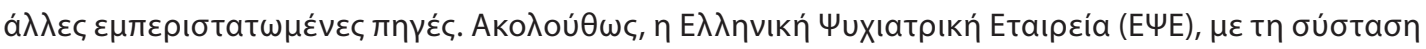

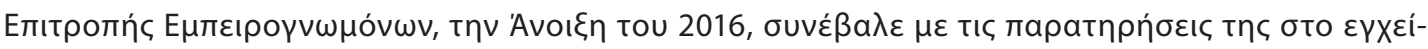

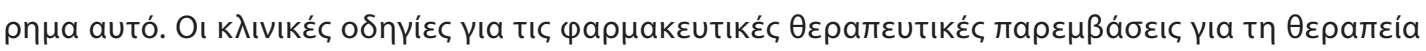

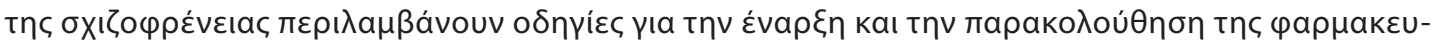

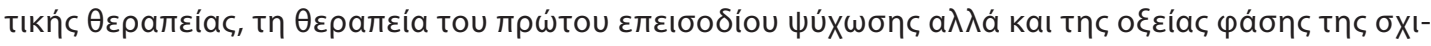

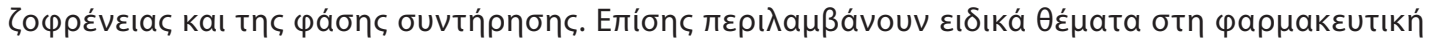

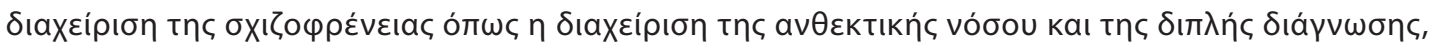




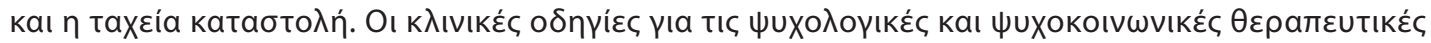

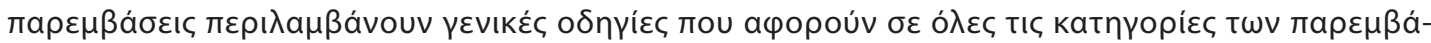

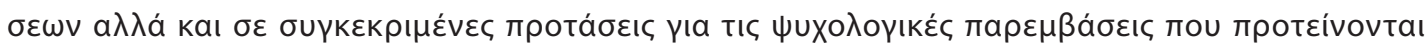

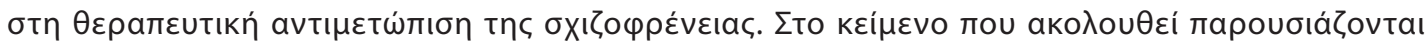

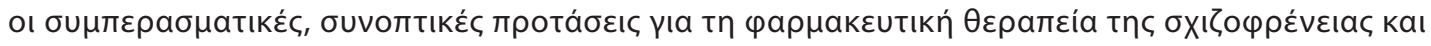

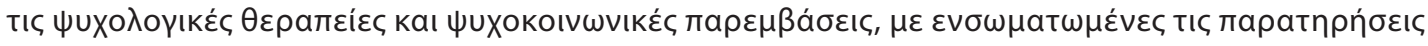
TIऽ E $\Psi E$.

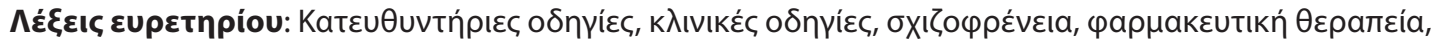

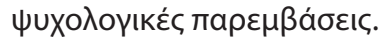

\section{Eıбaywyń}

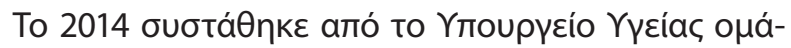

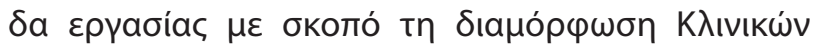

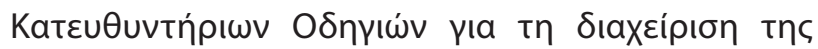

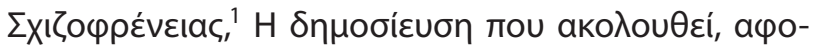

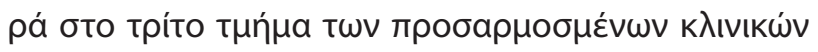

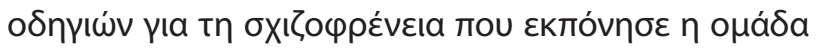

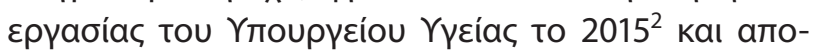

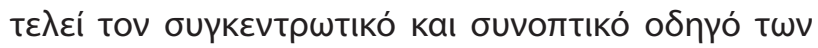

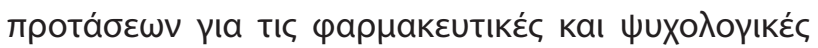

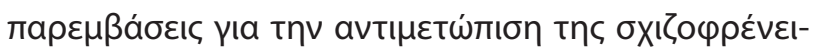

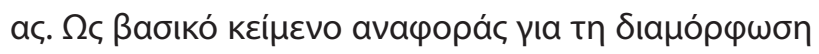

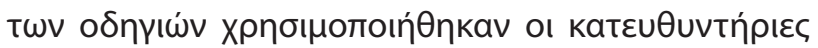

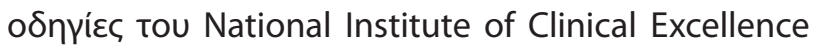

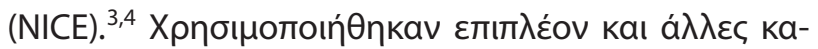

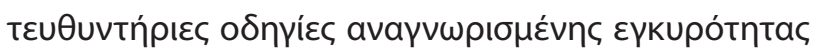

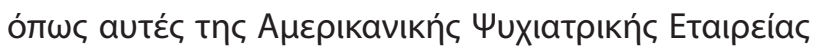
(American Psychiatric Association 2004), ${ }^{5}$ Tnc PORT

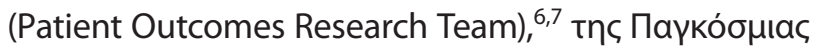

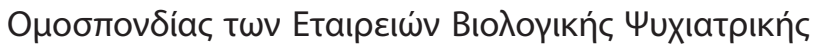
(World Federation of Societies of Biological Psychiatry,

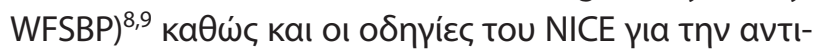

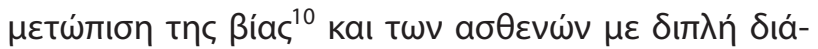

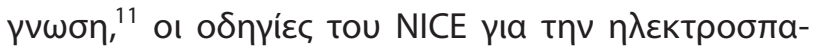

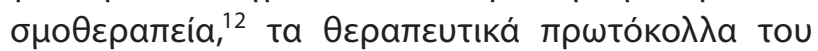

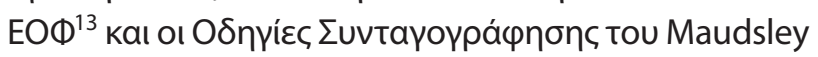

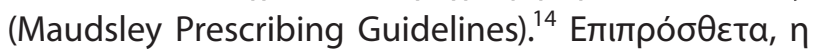

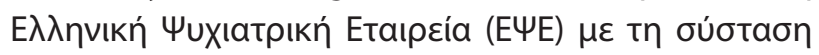

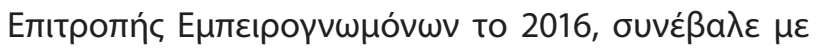

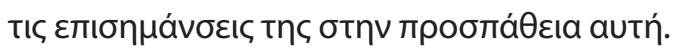

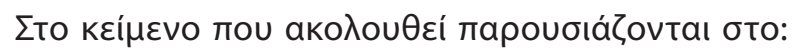

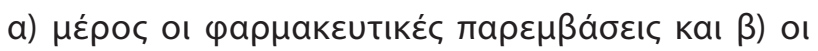

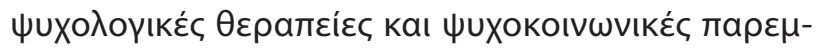

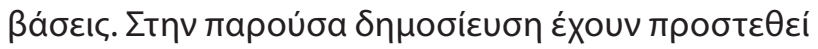

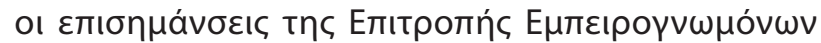
Tnऽ E $\Psi E$. ${ }^{1}$

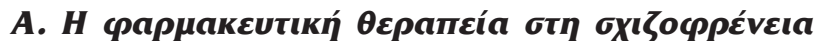

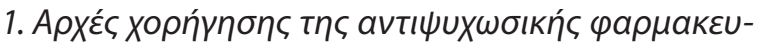

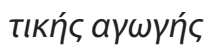

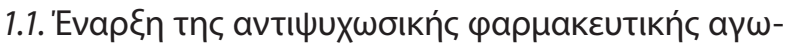
үńc.

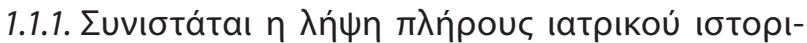
Koú.

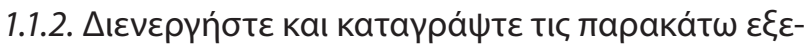

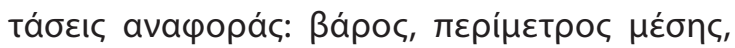

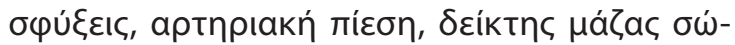

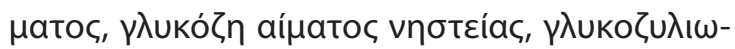

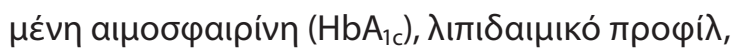

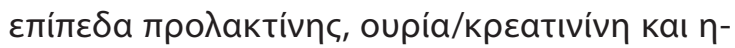

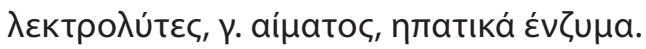

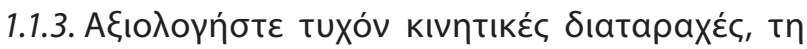

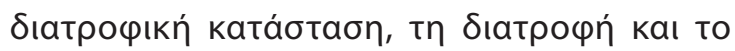

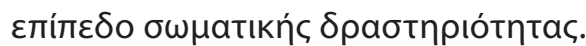

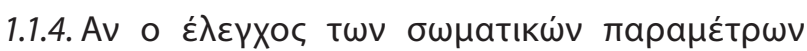

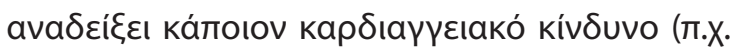

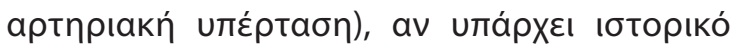

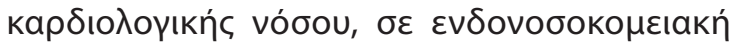

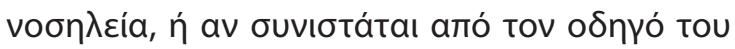

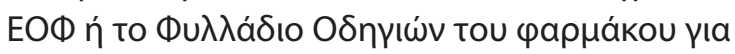

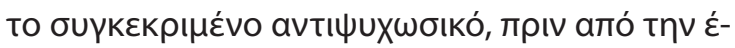

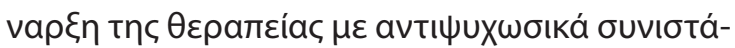

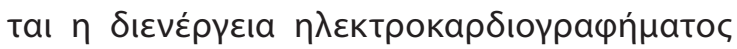
(НКГ).

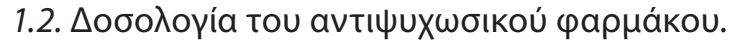

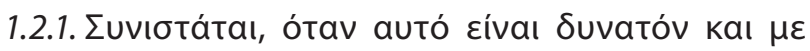

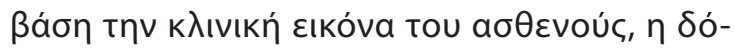

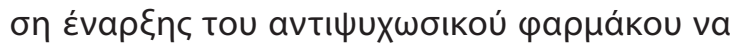




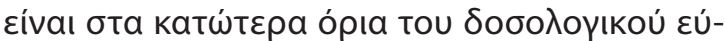

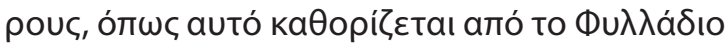

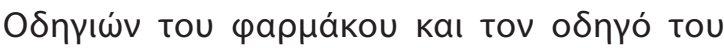
EOФ.

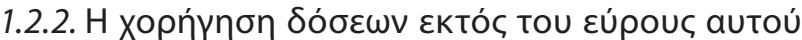

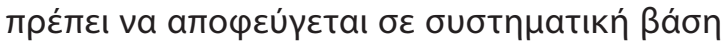

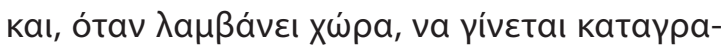

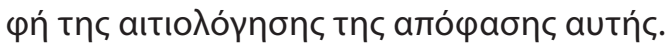

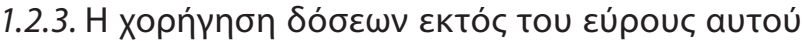

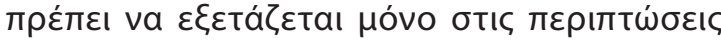

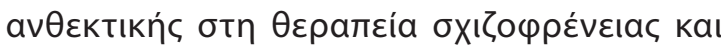

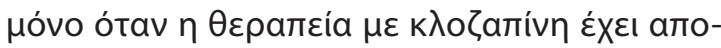

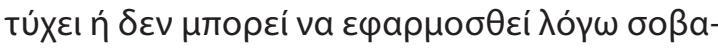

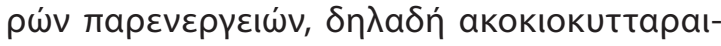

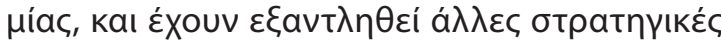

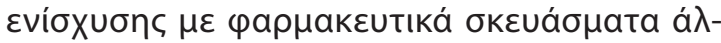

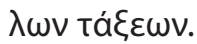

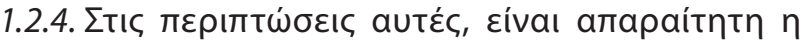

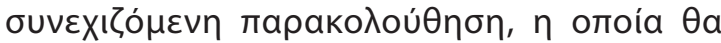

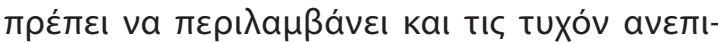
Өú

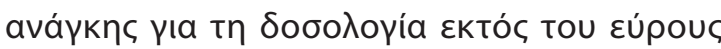

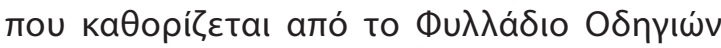

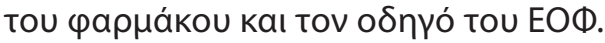

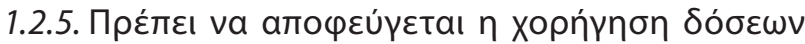

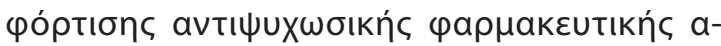
үwүńc.

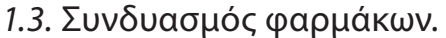

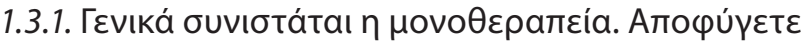

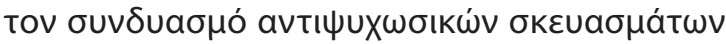

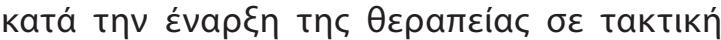

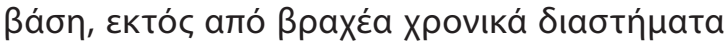

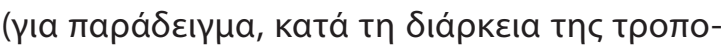

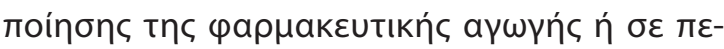

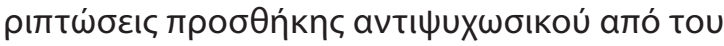

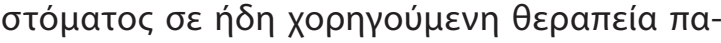

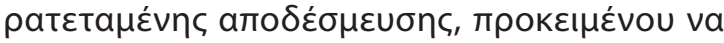

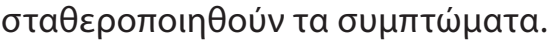

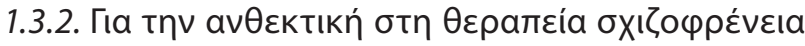

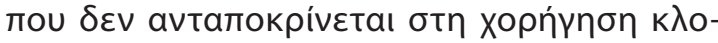

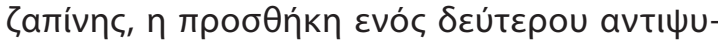

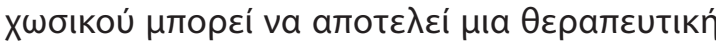

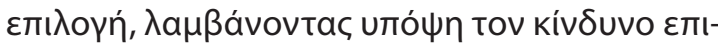

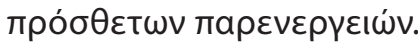

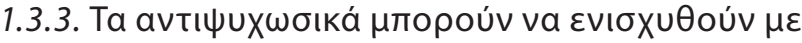

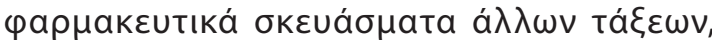

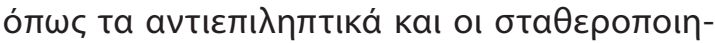

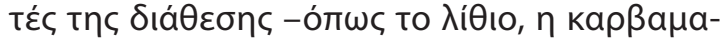

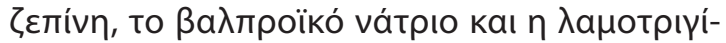

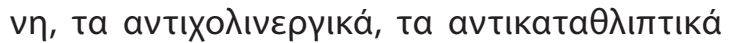

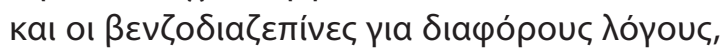

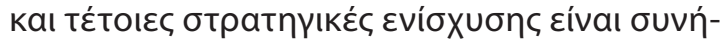

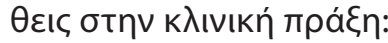

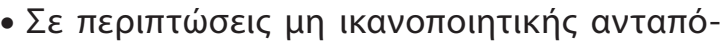

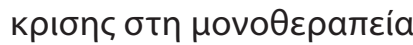

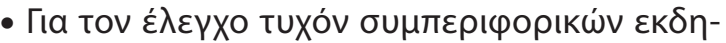
$\lambda \omega ́ \sigma \varepsilon \omega v$

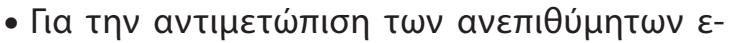

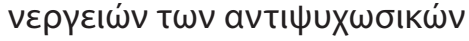

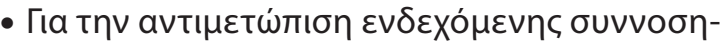

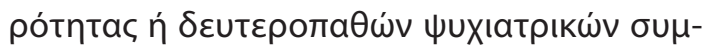

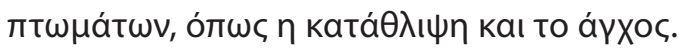

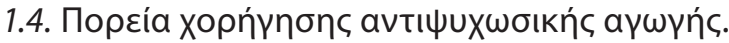

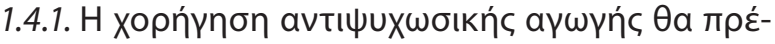

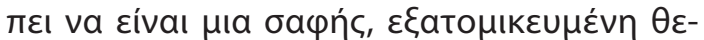

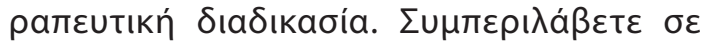

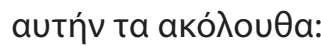

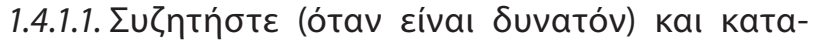

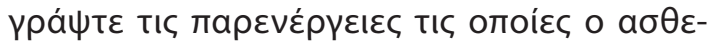

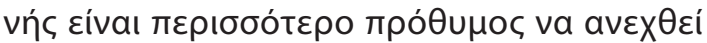

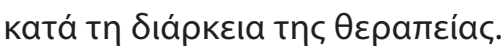

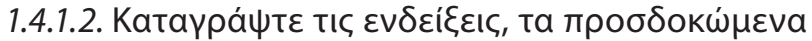

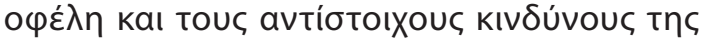

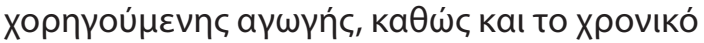

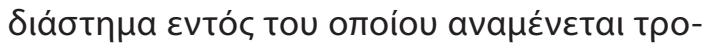

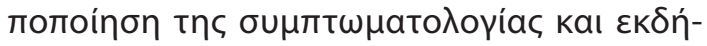

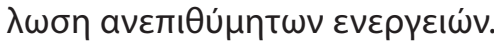

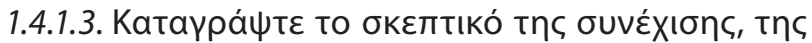

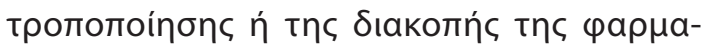

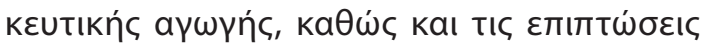

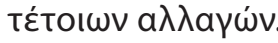

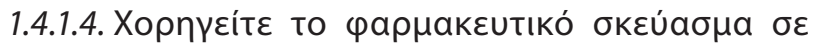

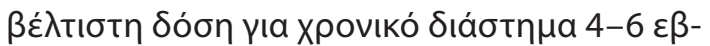
$\delta o \mu a ́ \delta \omega v$.

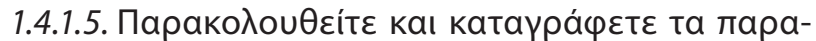

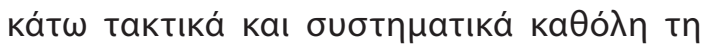

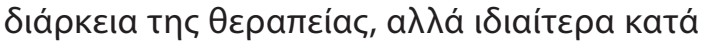

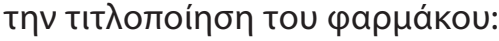

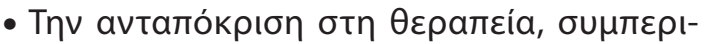

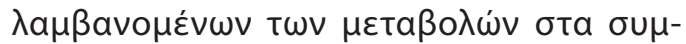

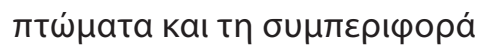

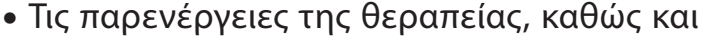

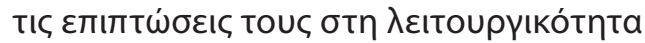




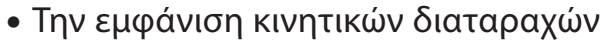

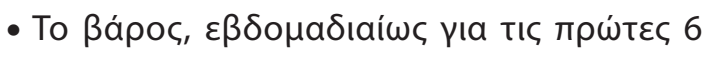

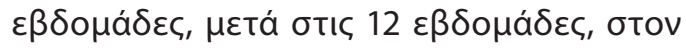

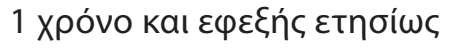

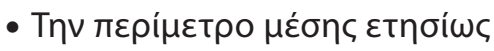

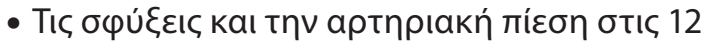

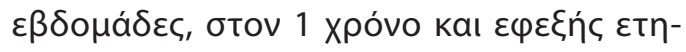

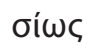

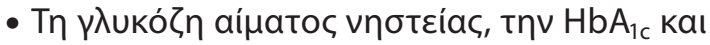

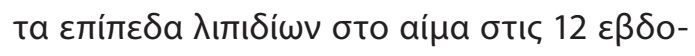

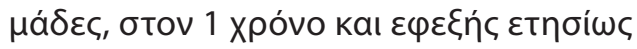

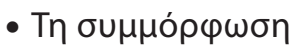

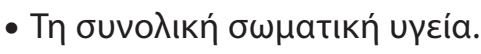

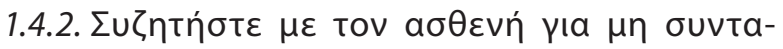

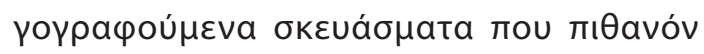

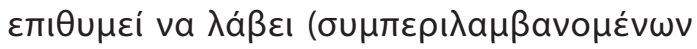

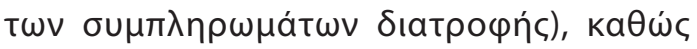

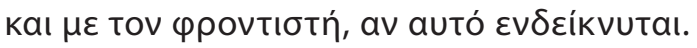

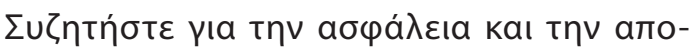

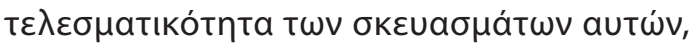

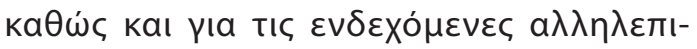

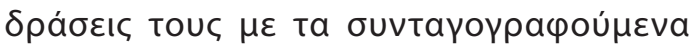

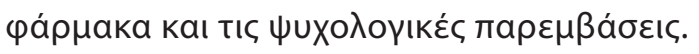

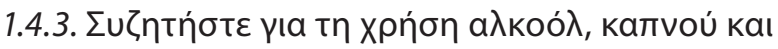

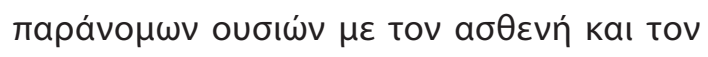

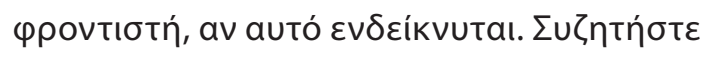

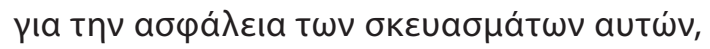

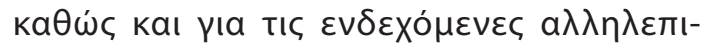

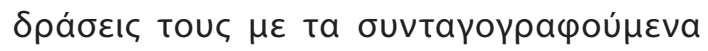

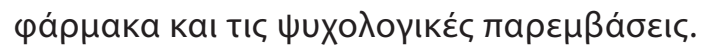

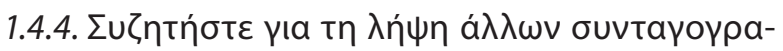

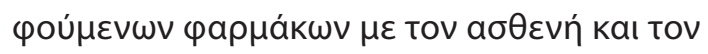

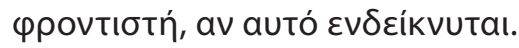

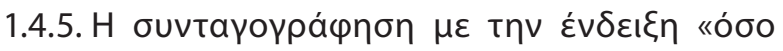

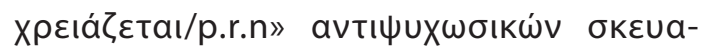

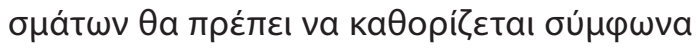

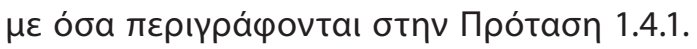

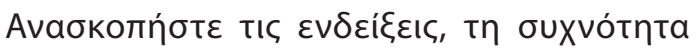

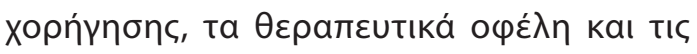

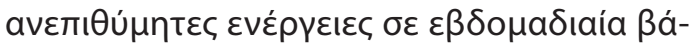

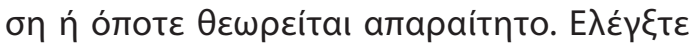

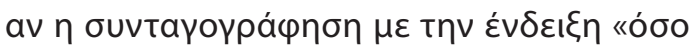

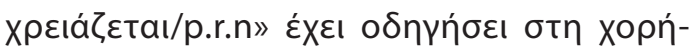

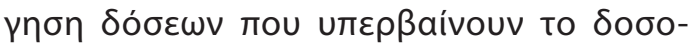

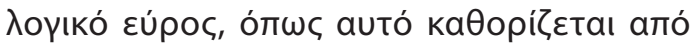

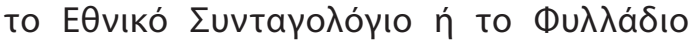

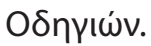

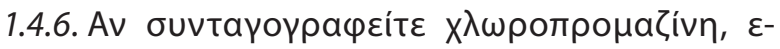

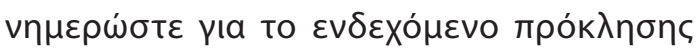

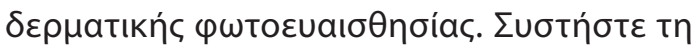

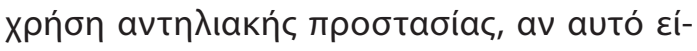
vaı amapaítnto.

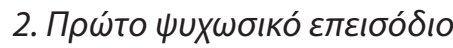

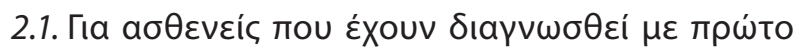

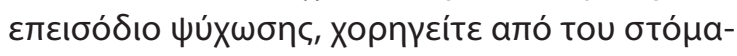

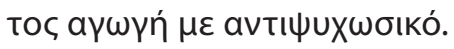

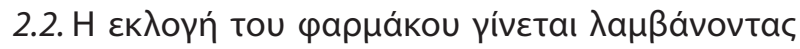

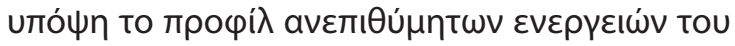

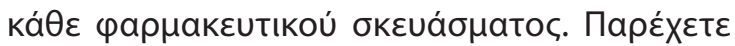

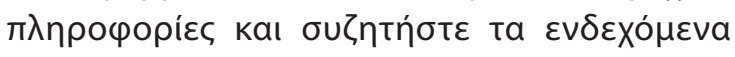

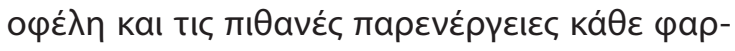

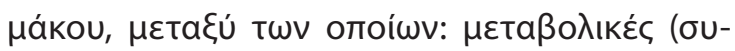

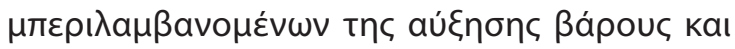

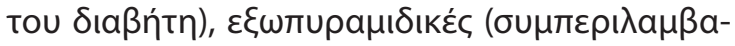

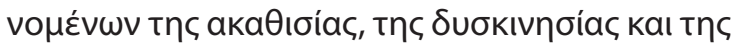

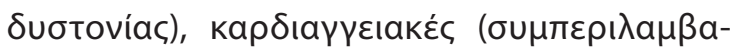

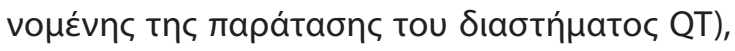

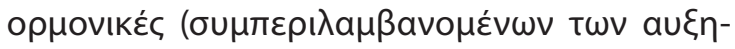

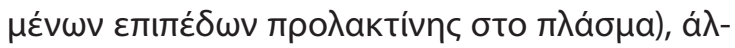

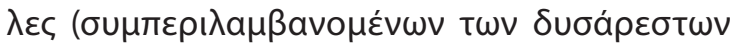

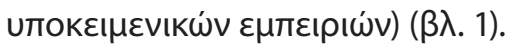

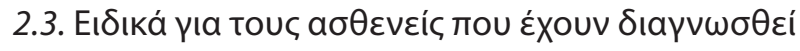

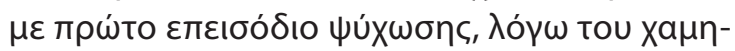

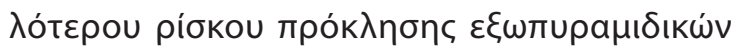

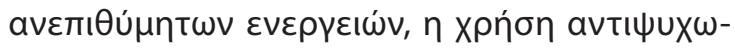

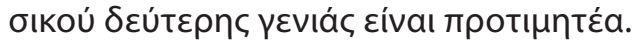

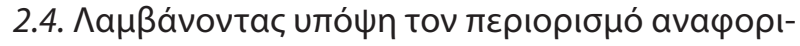

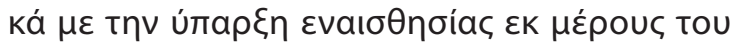

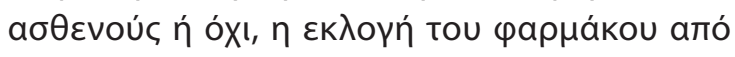

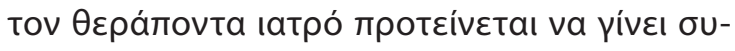

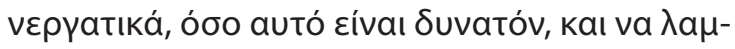

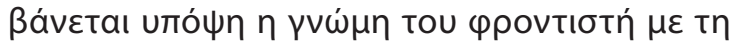

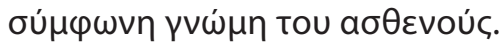

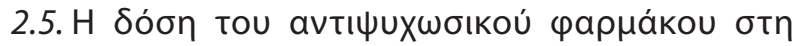

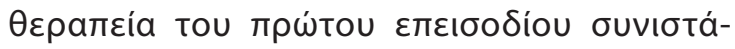

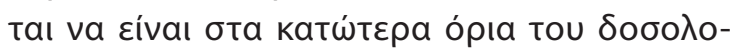

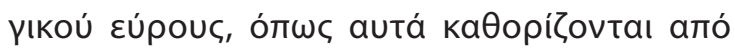

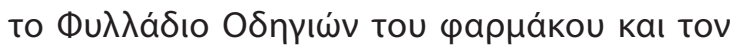

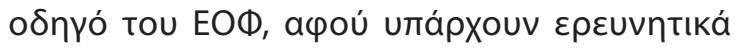

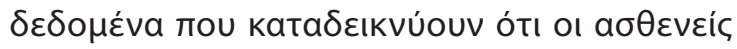




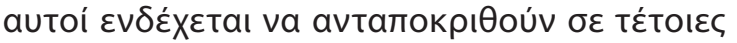

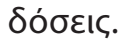

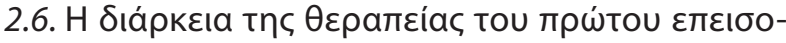

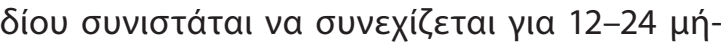

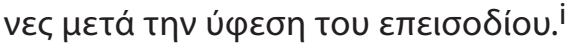

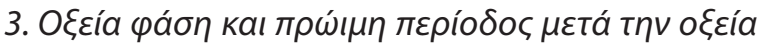

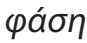

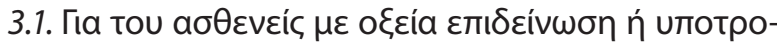

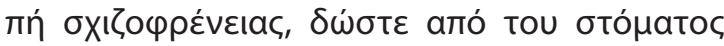

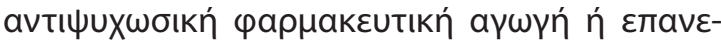

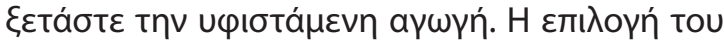

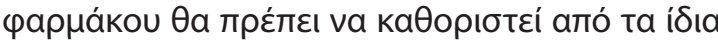

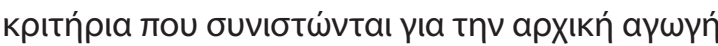

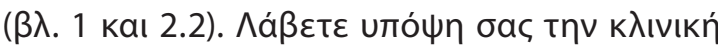

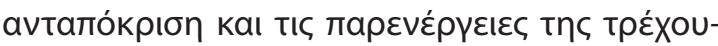

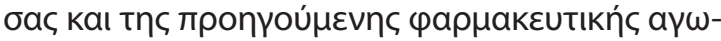

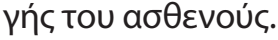

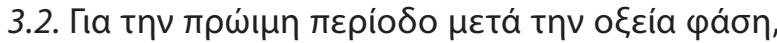

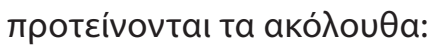

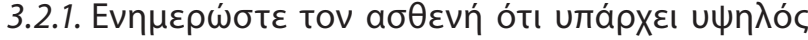

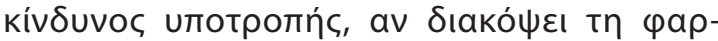

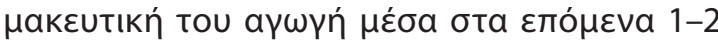
Xpóvia.

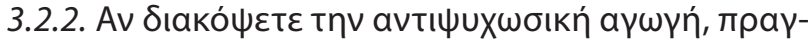

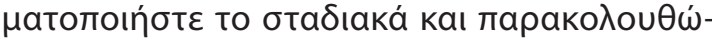

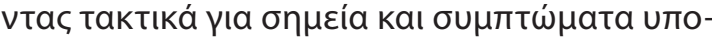
троти́c.

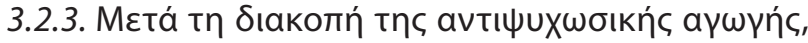

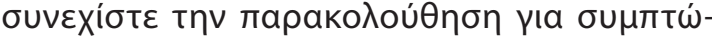

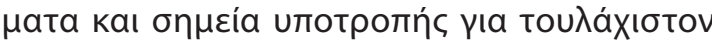
2 ह́tๆ.

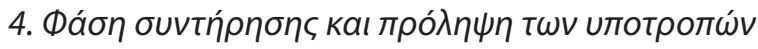

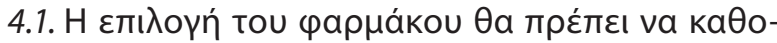

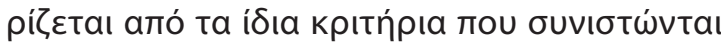

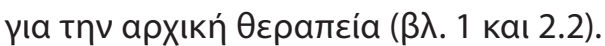

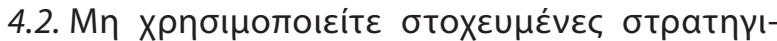

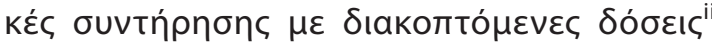

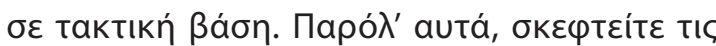

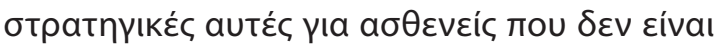

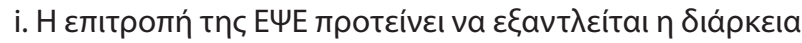
tnc 2-etíac.

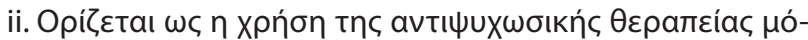

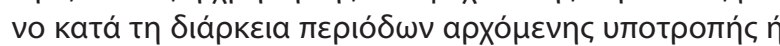

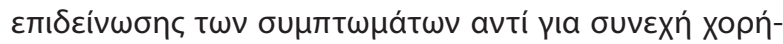
үnon.

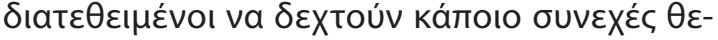

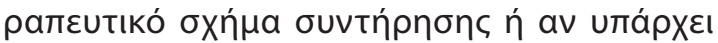

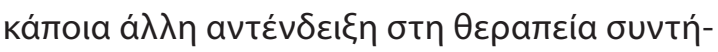

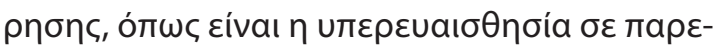

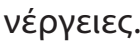

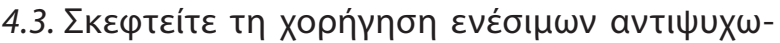

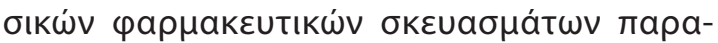

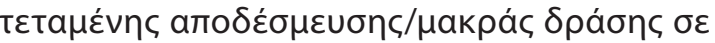

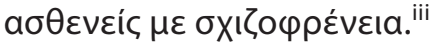

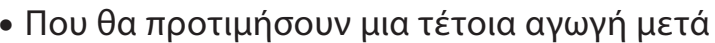

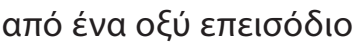

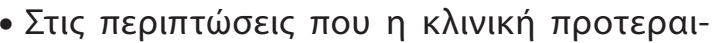

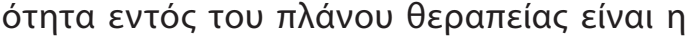

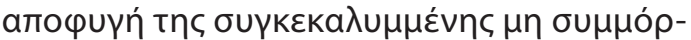

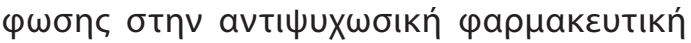
aүwүń.

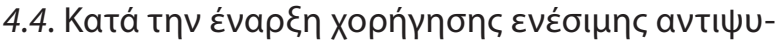

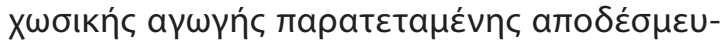
бП५:

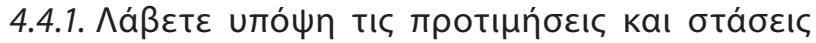

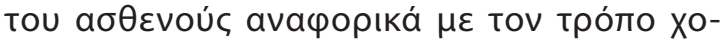

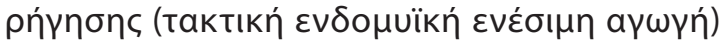

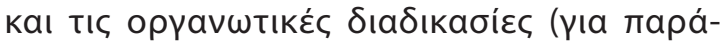

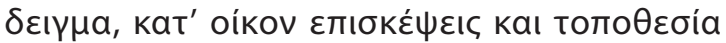

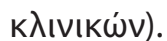

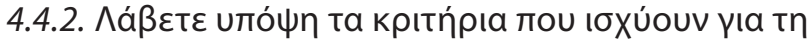

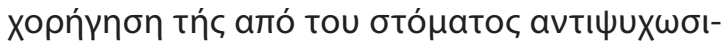

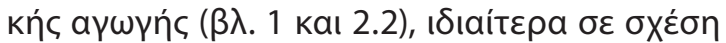

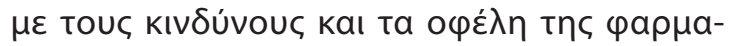

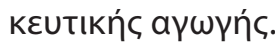

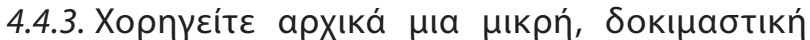

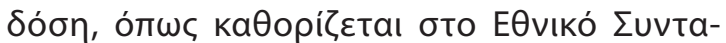

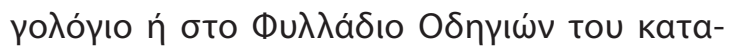

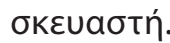

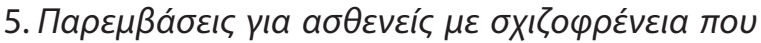

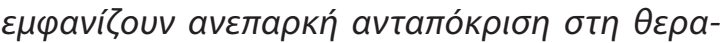

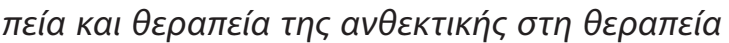

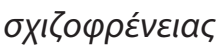

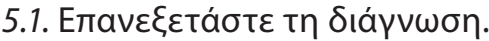

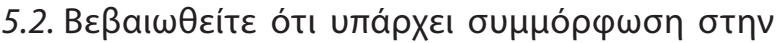

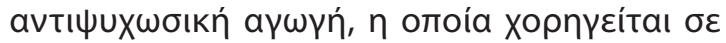

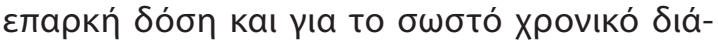
бтпна.

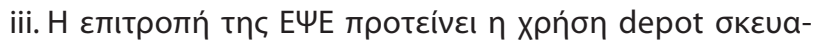

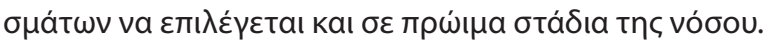




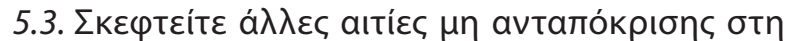

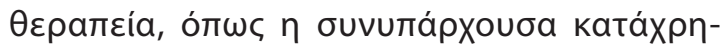

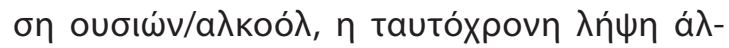

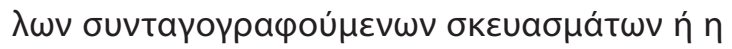

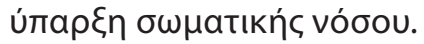

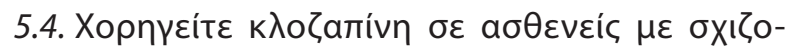

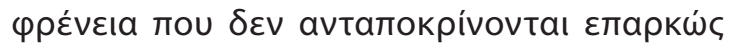

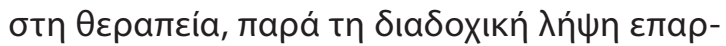

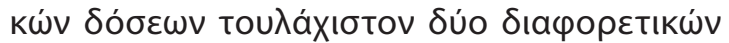

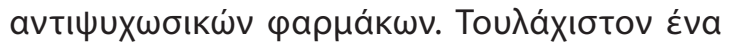

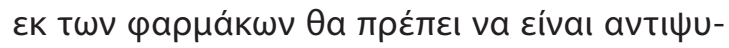

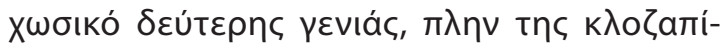

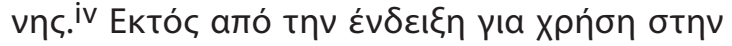

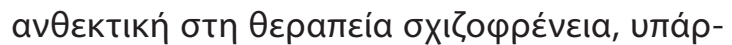

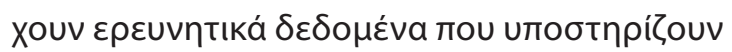

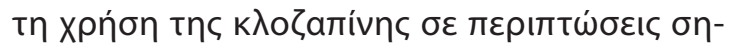

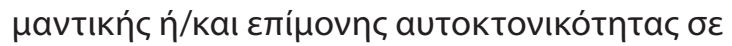

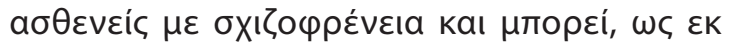

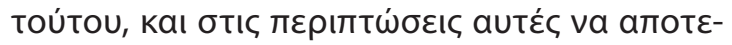

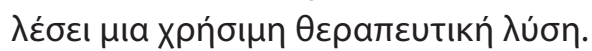

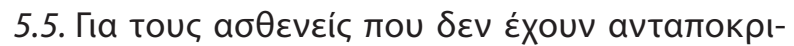

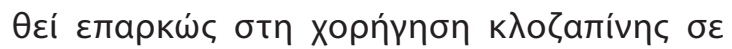

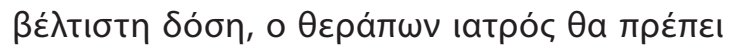

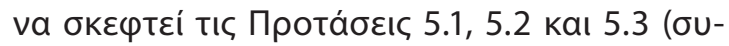

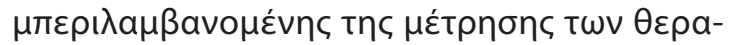

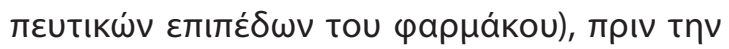

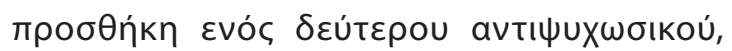

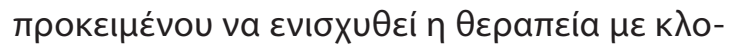

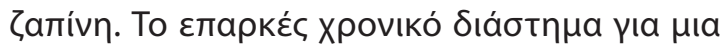

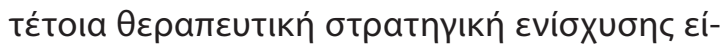

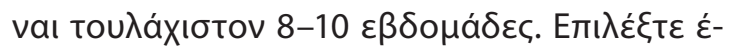

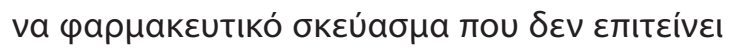

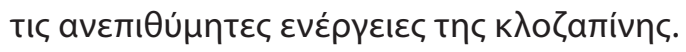

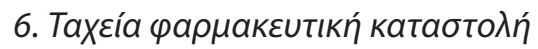

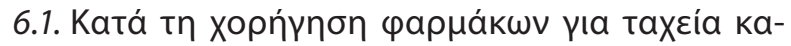
табто入и́:

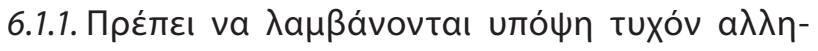

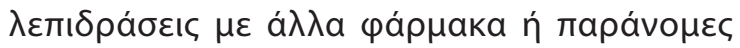

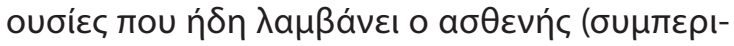

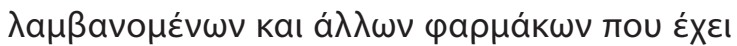

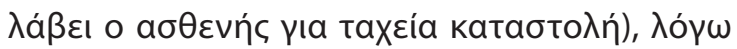

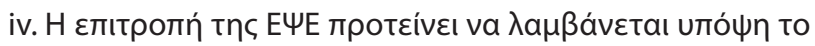

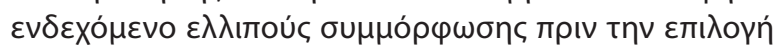

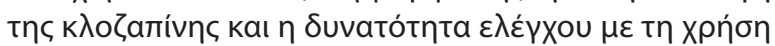

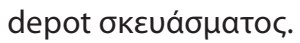

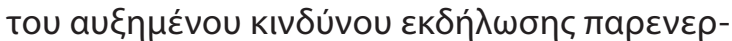

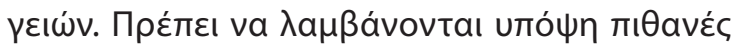

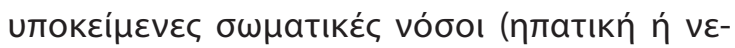

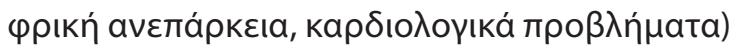
ท́ $\varepsilon ү K \cup \mu о \sigma u ́ v \eta$.

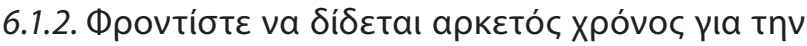

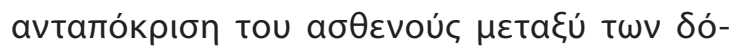

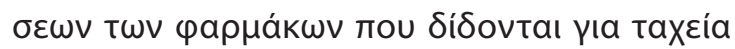
катабто入и́.

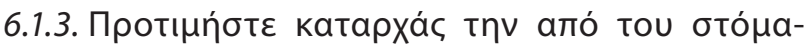

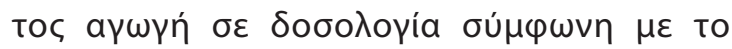

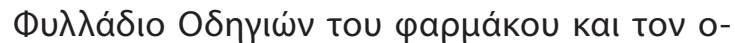

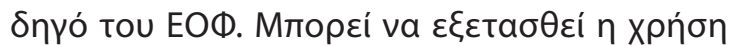

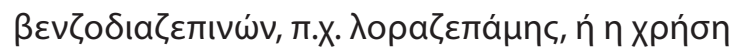

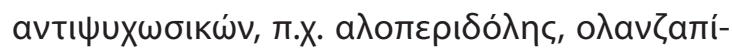

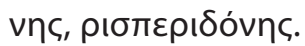

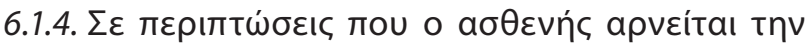

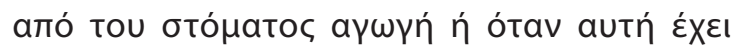

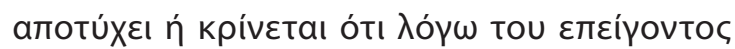

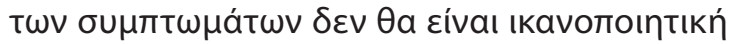

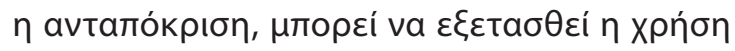

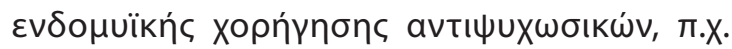

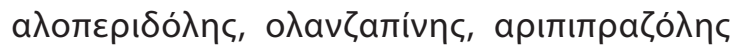

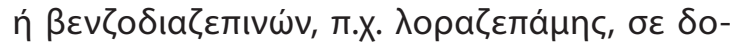

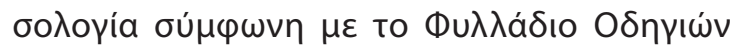

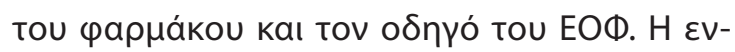

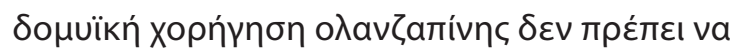

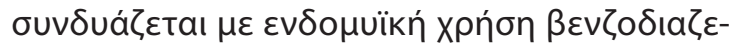

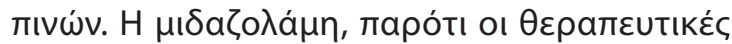

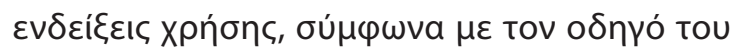

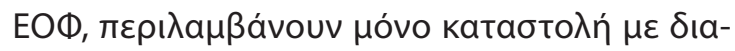

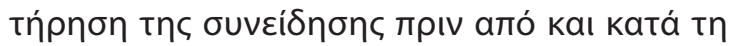

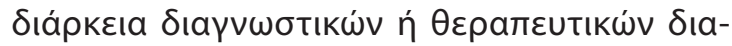

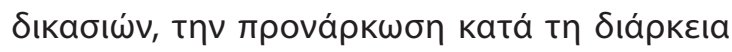

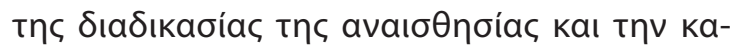

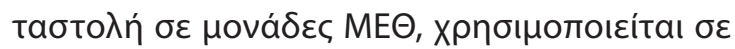

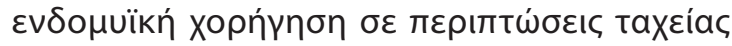

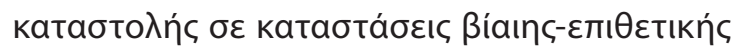

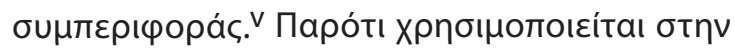

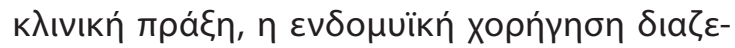

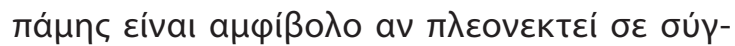

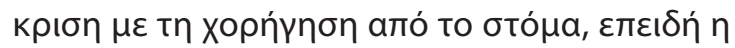

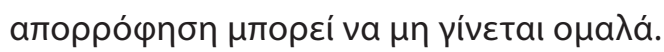

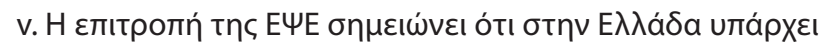

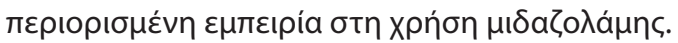




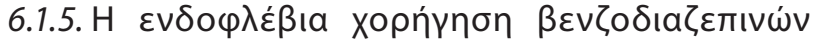

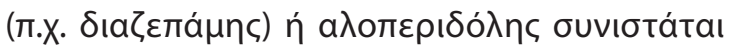

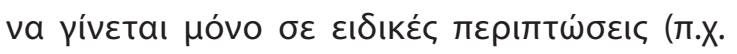

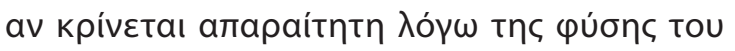

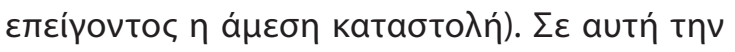

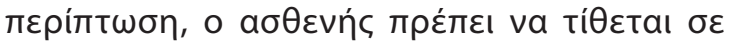

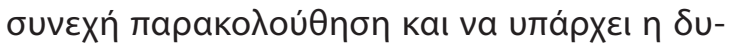

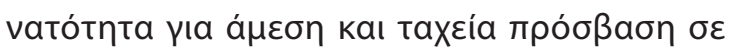

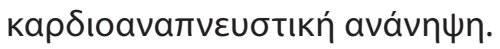

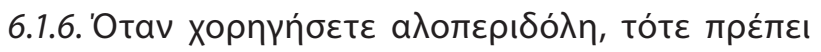

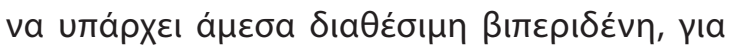

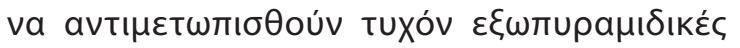

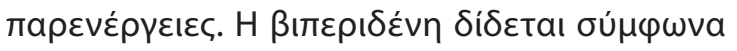

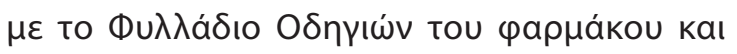

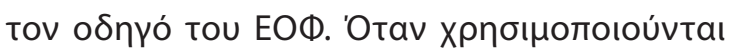

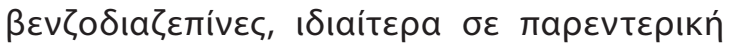

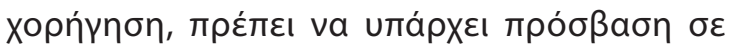

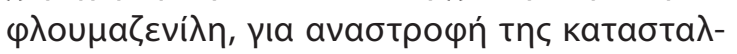

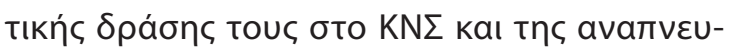

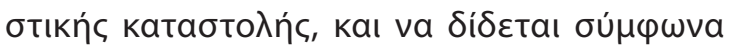

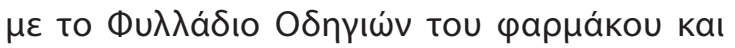

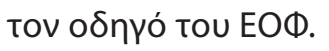

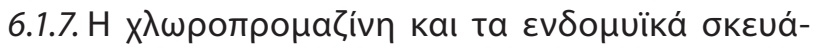

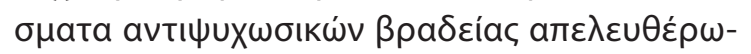

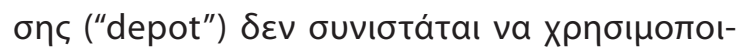

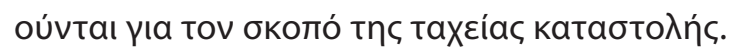

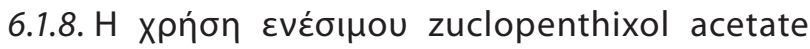

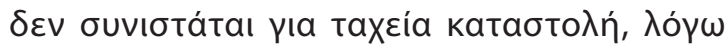

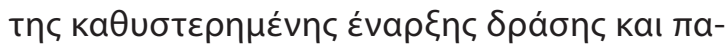

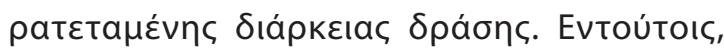

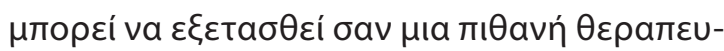

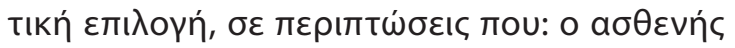

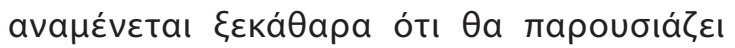

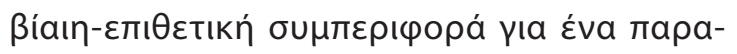

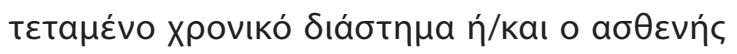

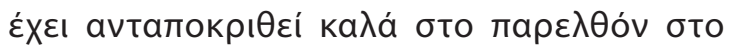

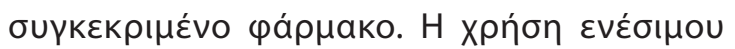

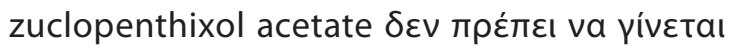

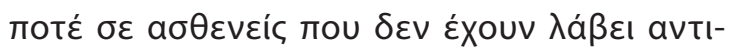

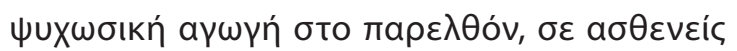

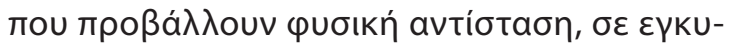

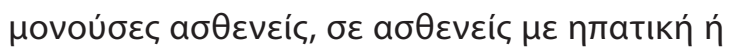

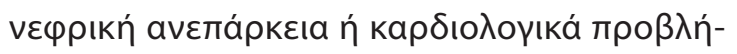

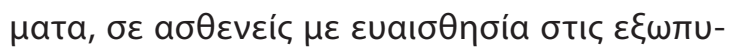

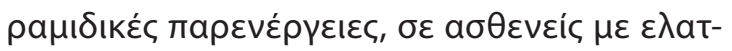

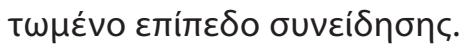

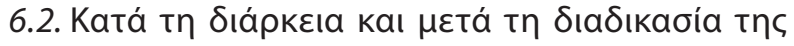

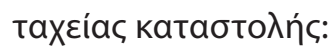

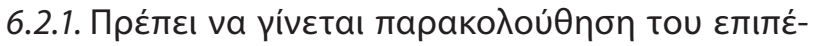

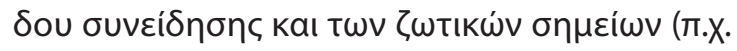

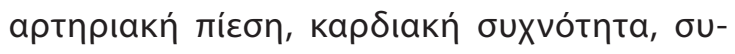

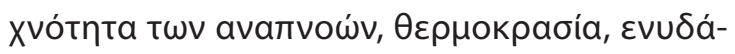

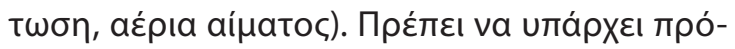

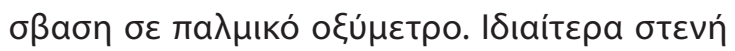

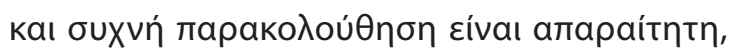

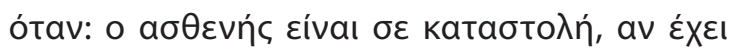

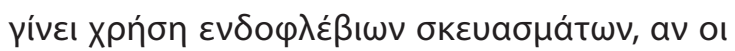

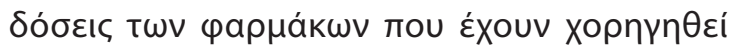

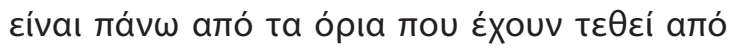

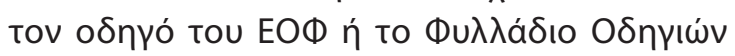

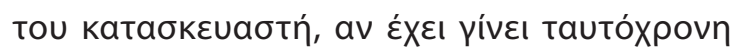

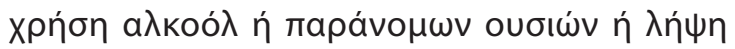

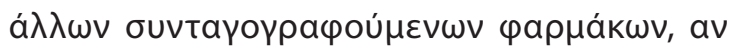

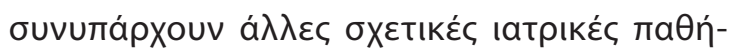
бहाৎ.

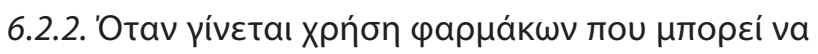

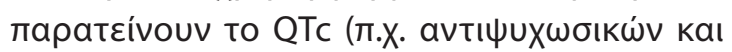

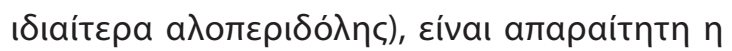

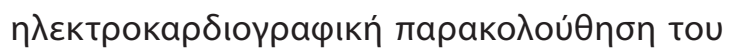

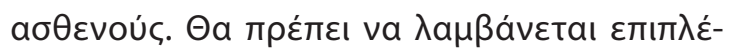

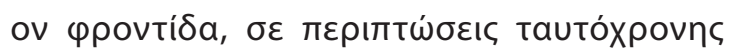

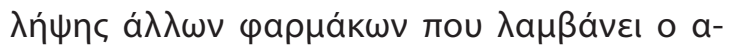

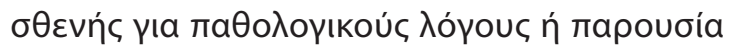

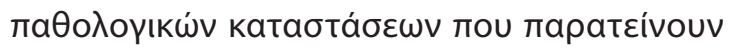
To QTC.

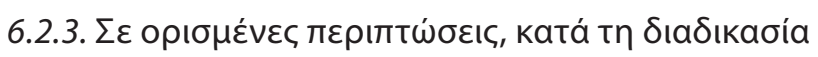

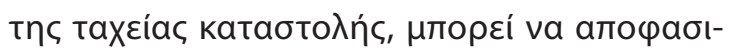

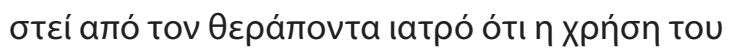

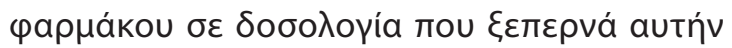

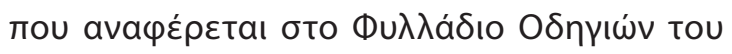

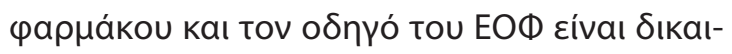

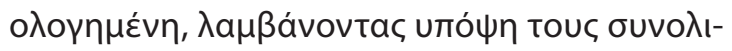

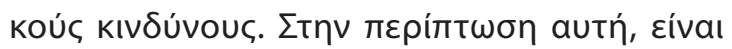

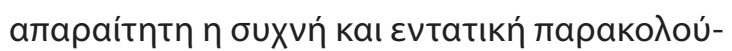

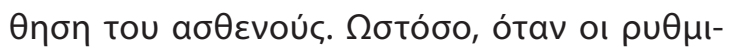

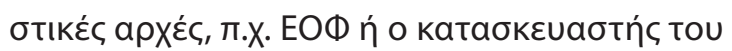

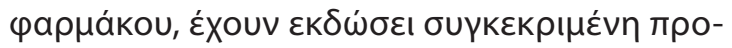

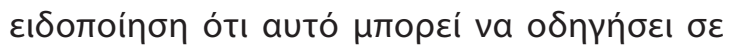

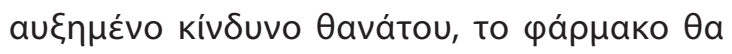

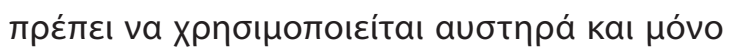

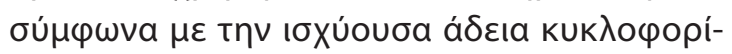
ac otnv aүopá. 


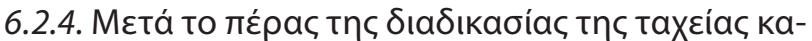

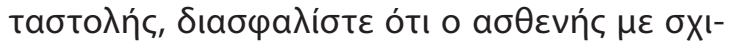

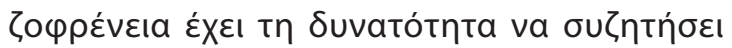

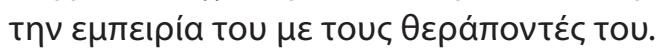

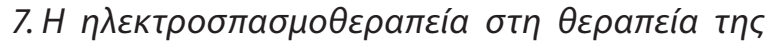

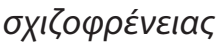

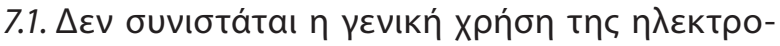

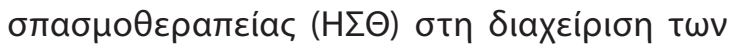

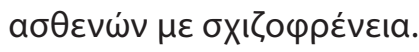

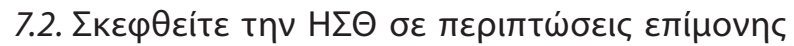

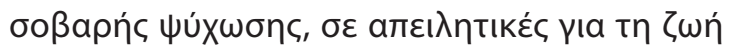

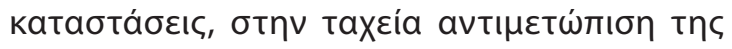

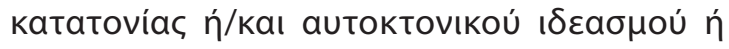

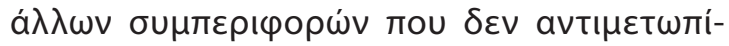

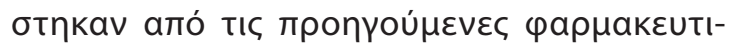

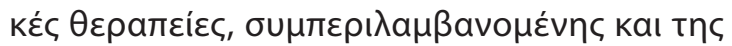

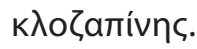

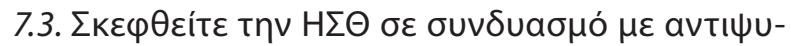

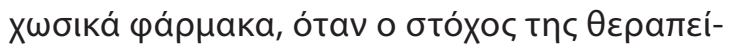

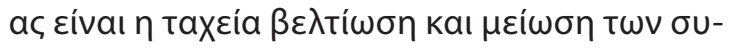

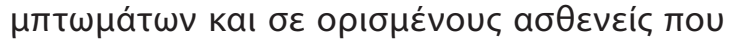

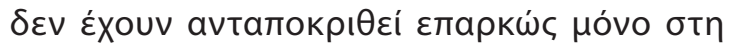

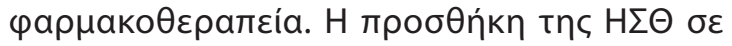

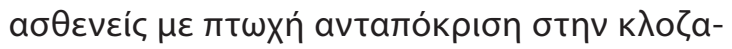

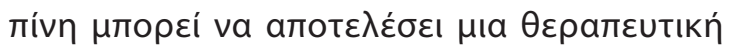

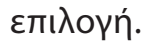

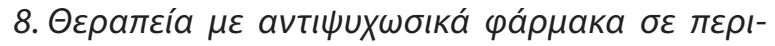

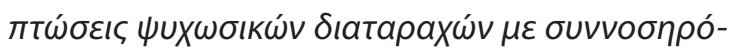

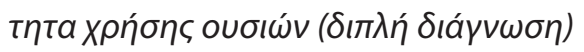

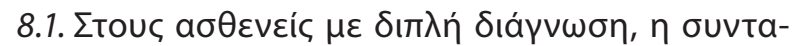

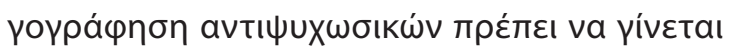

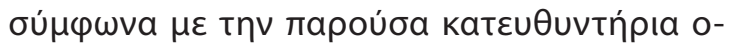

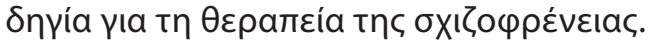

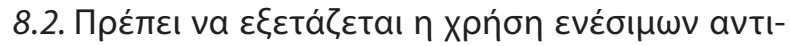

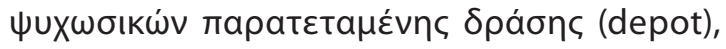

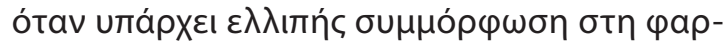

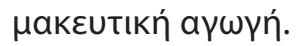

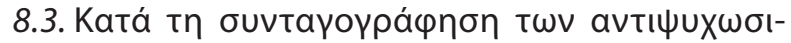

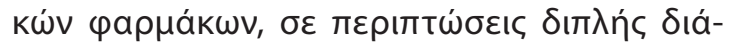

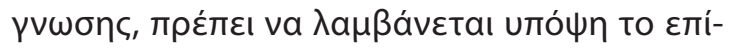

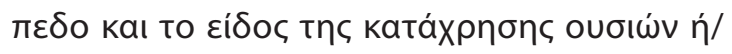

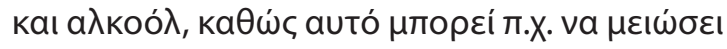

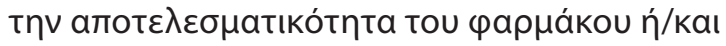

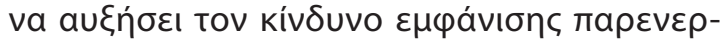

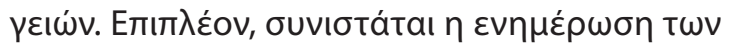

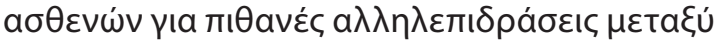

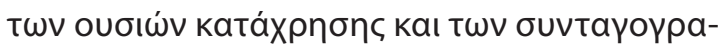

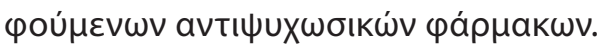

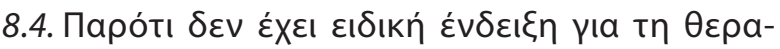

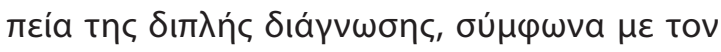

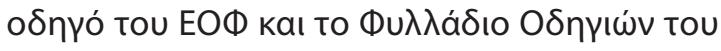

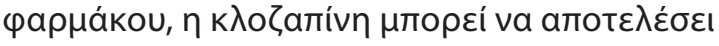

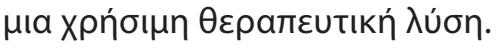

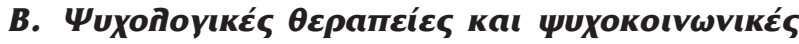

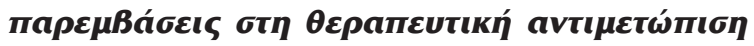

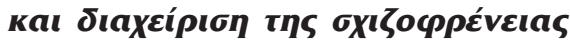

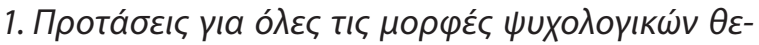

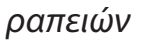

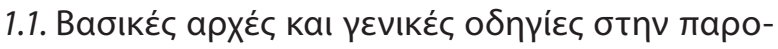

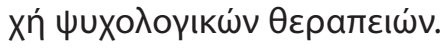

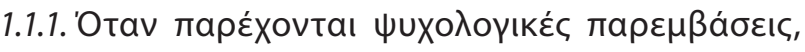

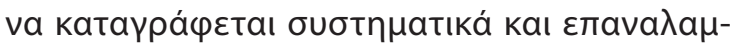

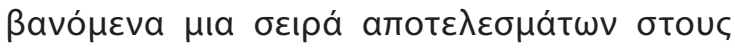

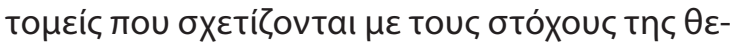

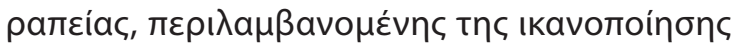

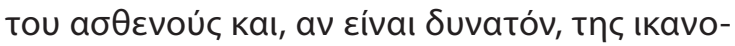

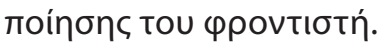

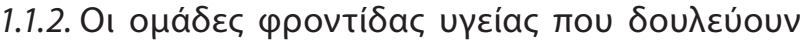

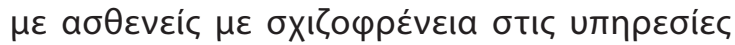

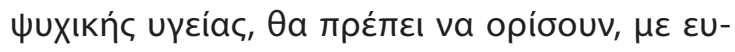

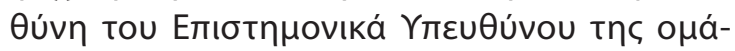

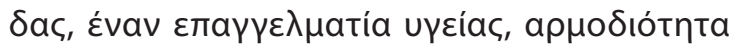

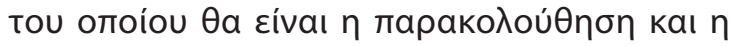
avaбко́тпбп:

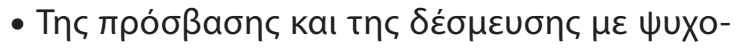

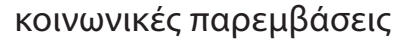

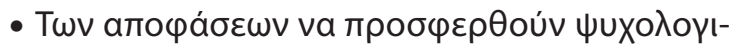

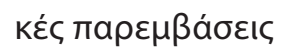

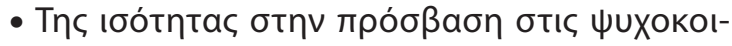

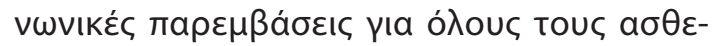

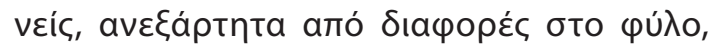

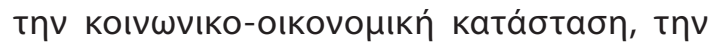

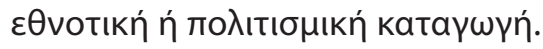

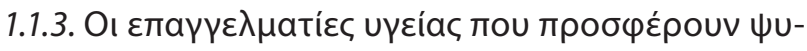

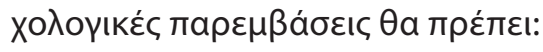

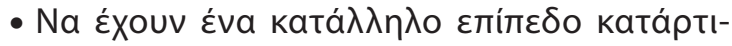

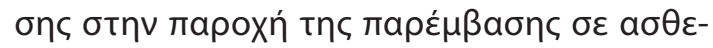

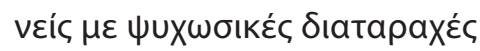

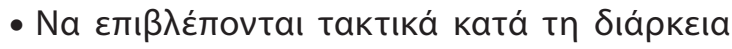

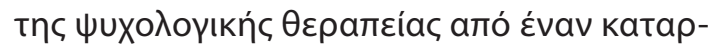




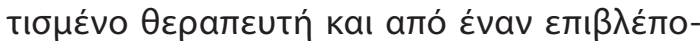
vта-єпо́ттп.

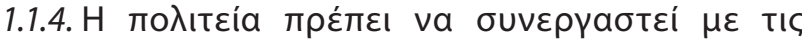

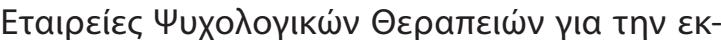

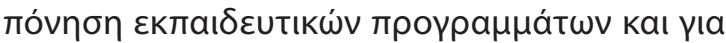

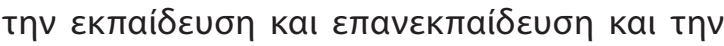

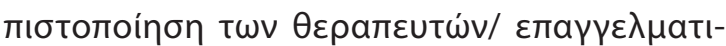

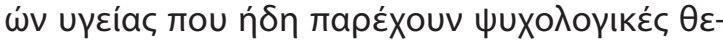

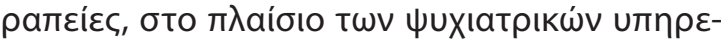

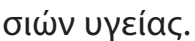

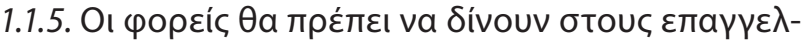

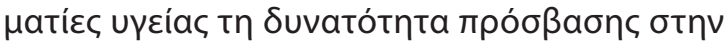

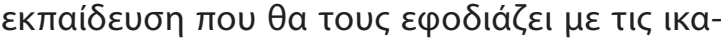

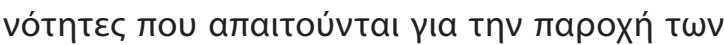

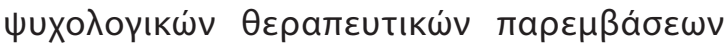

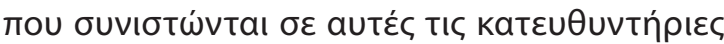

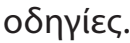

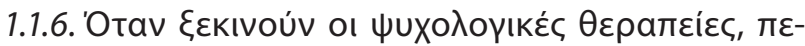

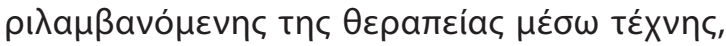

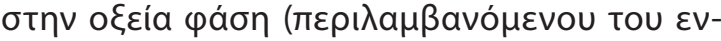

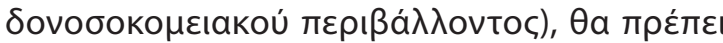

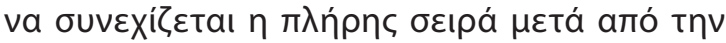

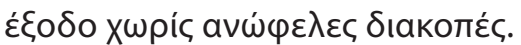

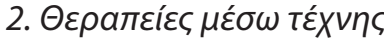

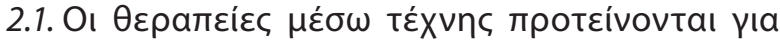

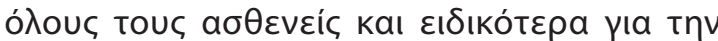

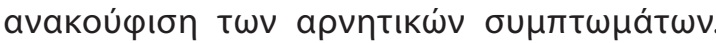

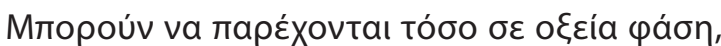

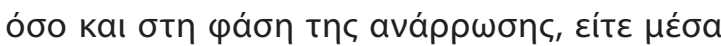

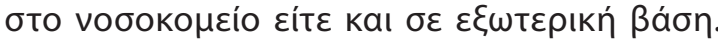

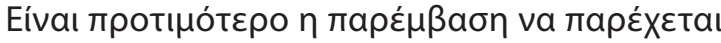

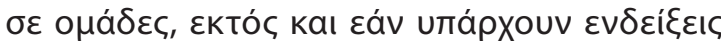
үıа то avtí日eto.

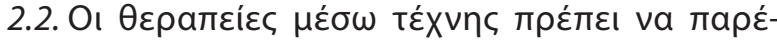

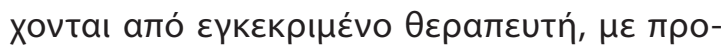

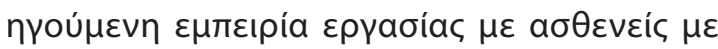

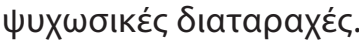

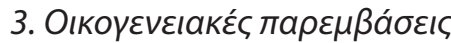

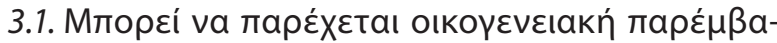

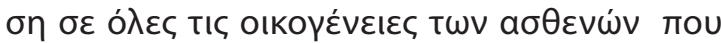

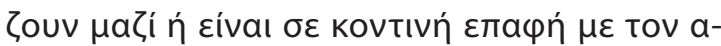

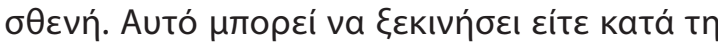

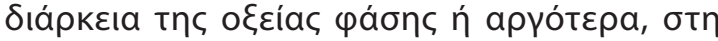

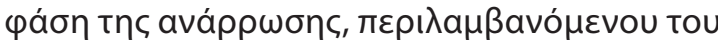

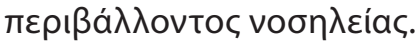

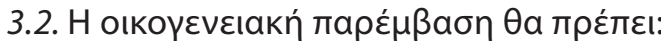

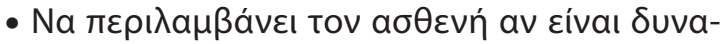
tóv

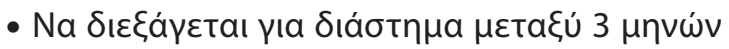
kaı 1 ह́tous

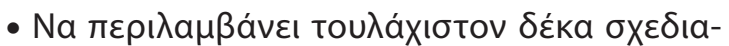

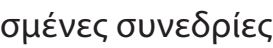

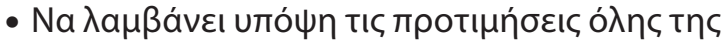

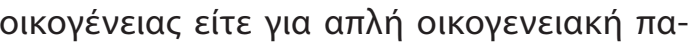

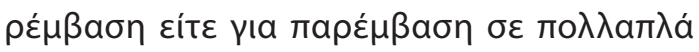

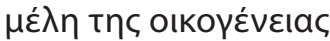

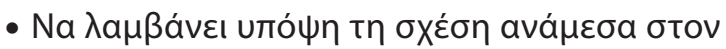
кúpı

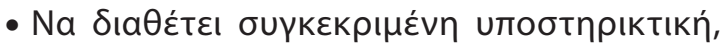

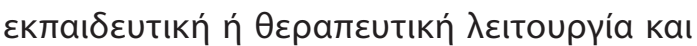

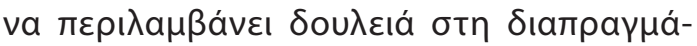

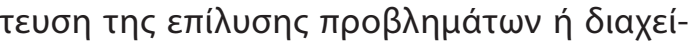

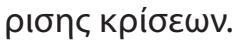

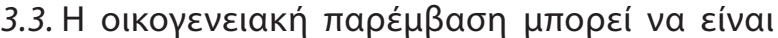

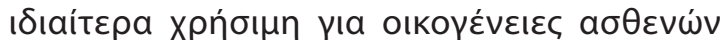
поO ह́Xouv:

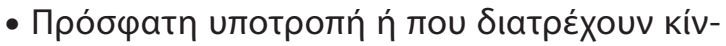

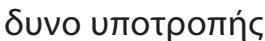

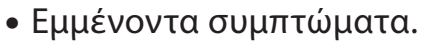

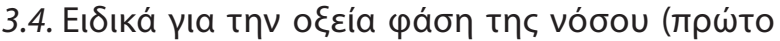

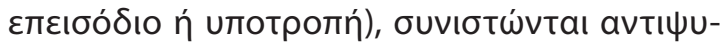

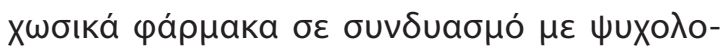

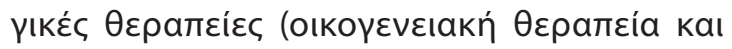

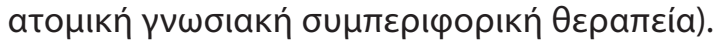

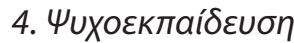

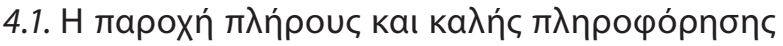

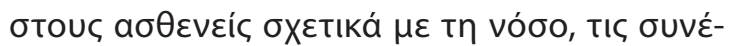

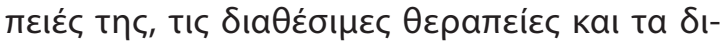

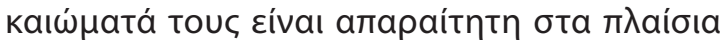

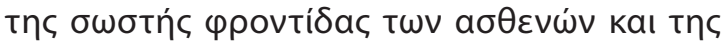

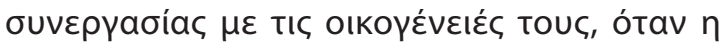

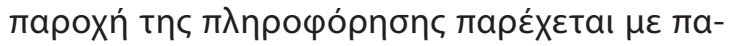

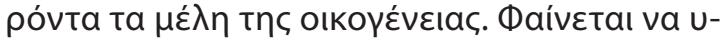

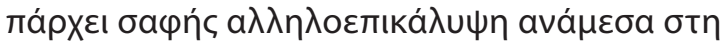

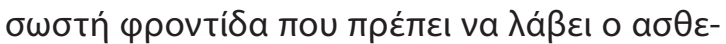

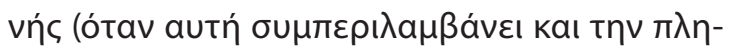

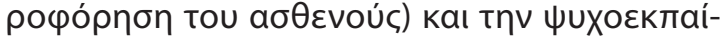

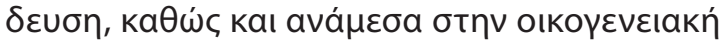

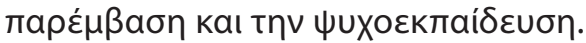




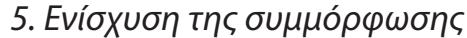

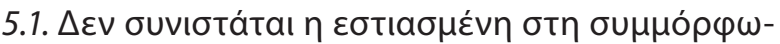

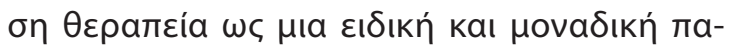

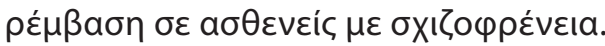

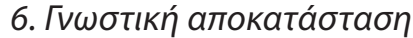

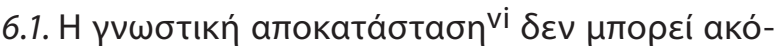

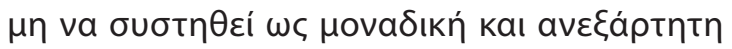

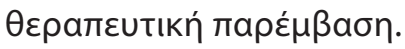

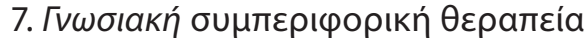

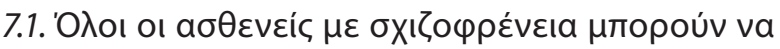

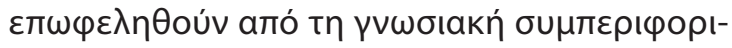

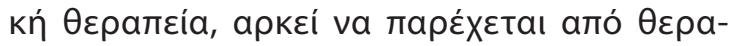

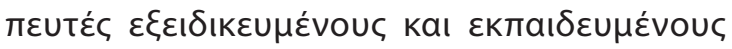

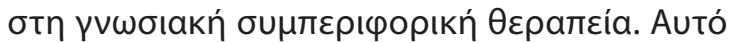

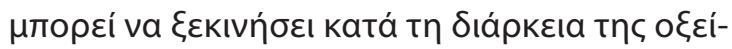

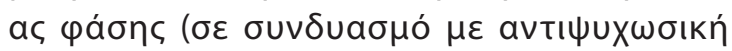

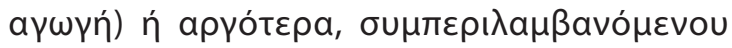

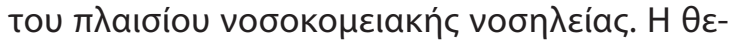

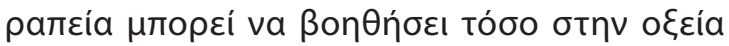

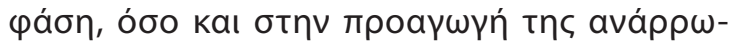

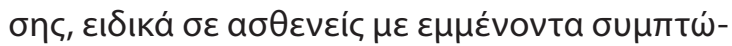

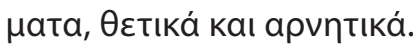

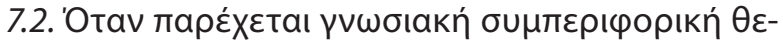

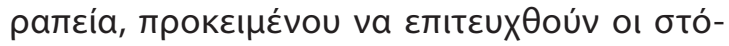

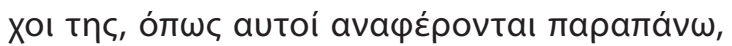

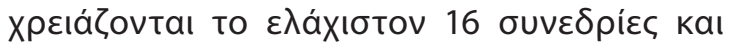

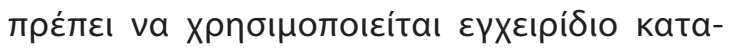

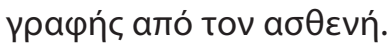

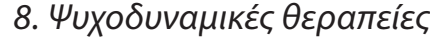

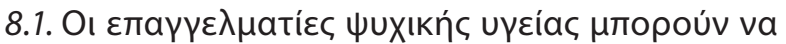

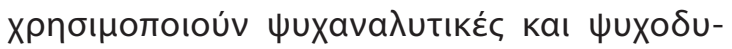

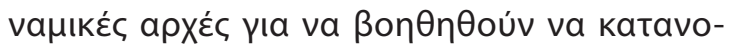

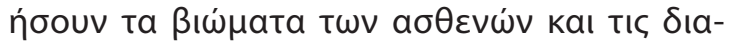

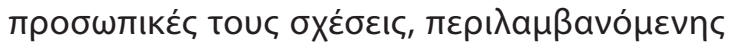

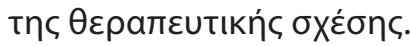

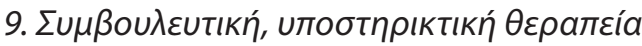

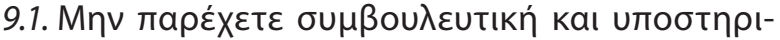

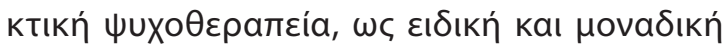

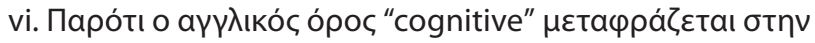

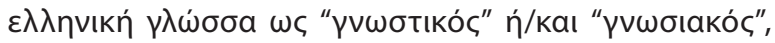

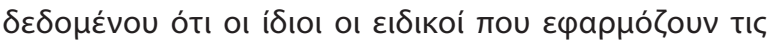

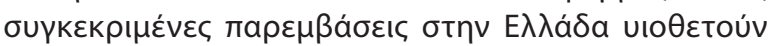

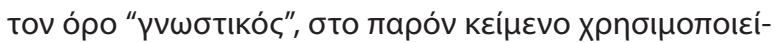

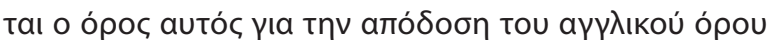

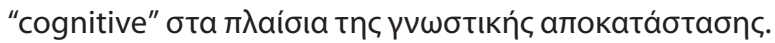

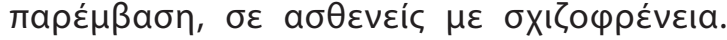

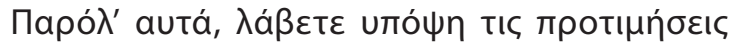

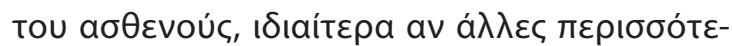

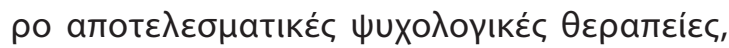

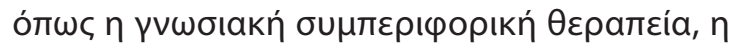

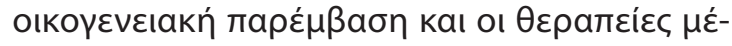

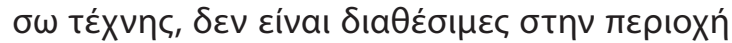
oac.

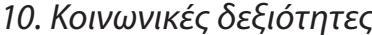

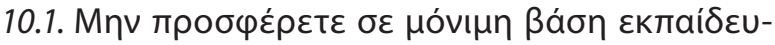

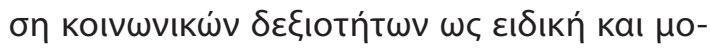

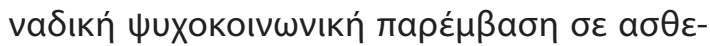

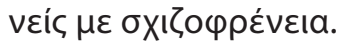

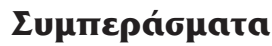

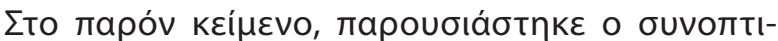

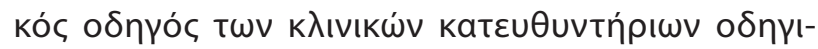

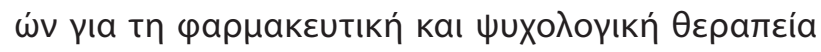

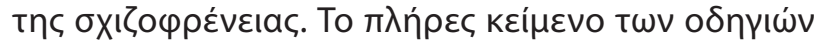

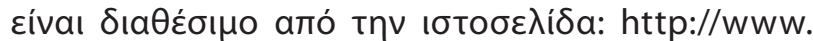

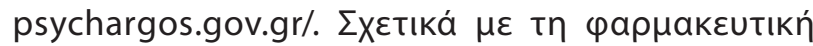

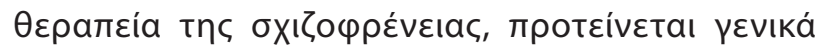

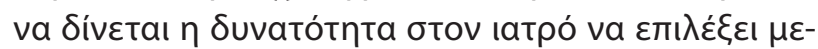

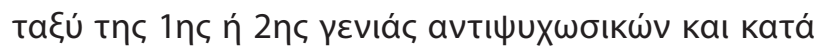

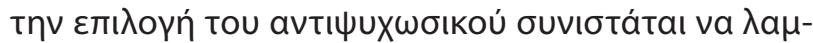

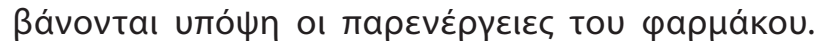

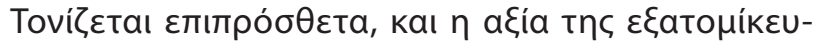

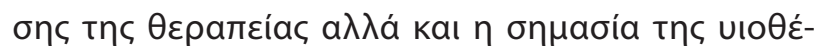

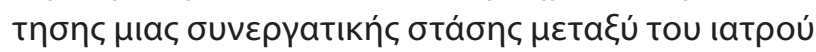

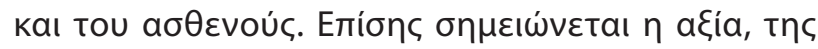

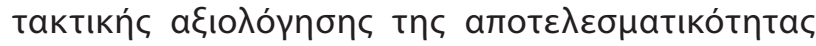

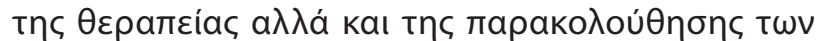

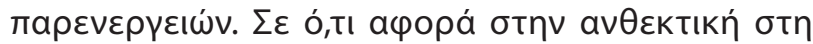

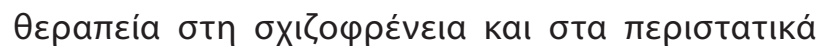

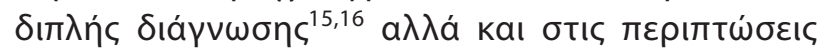

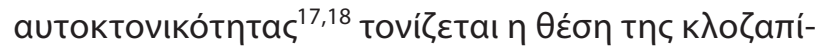

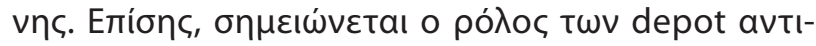

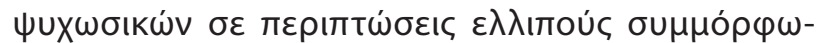

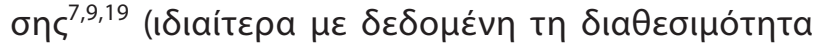

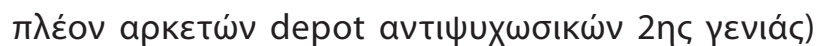

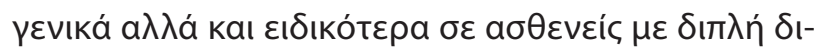

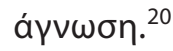

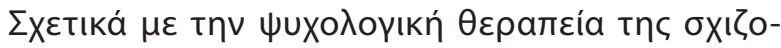

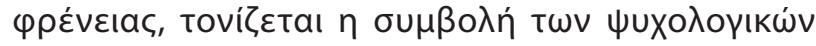

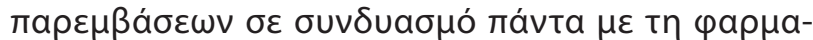

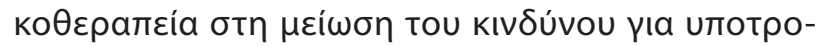




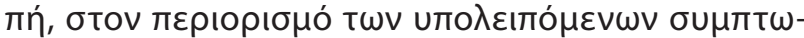

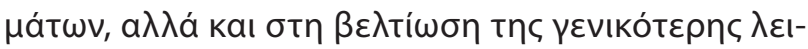

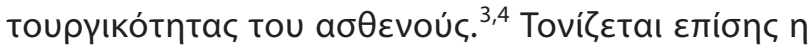

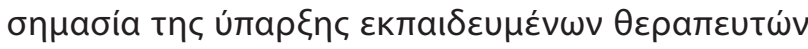

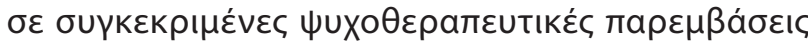

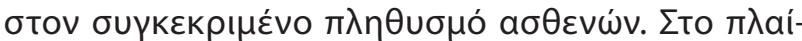

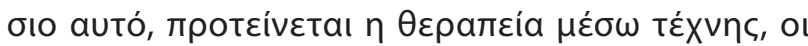

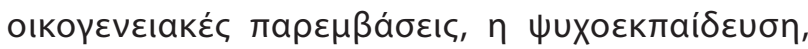

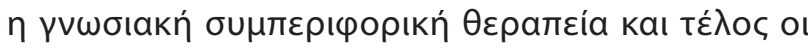

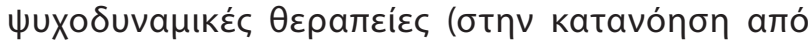

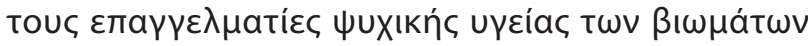

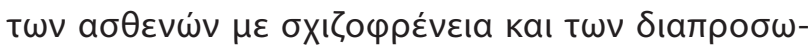

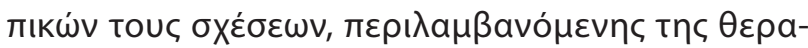

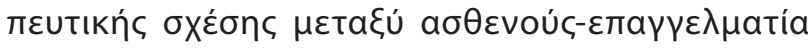

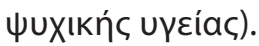

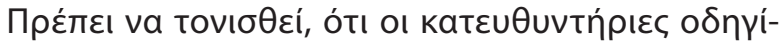

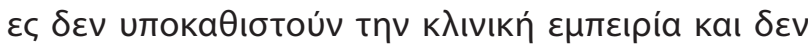

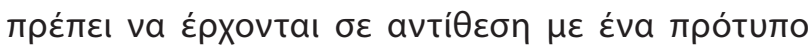

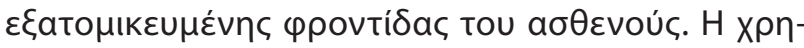

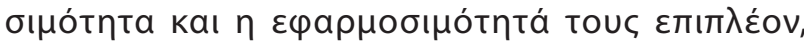

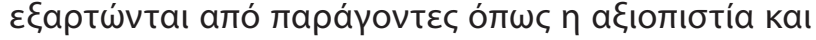

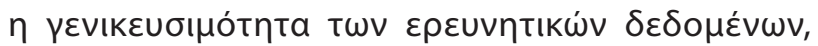

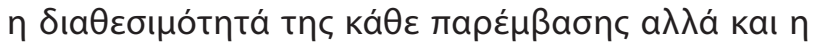

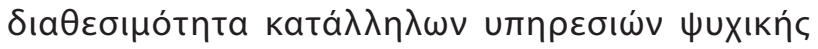

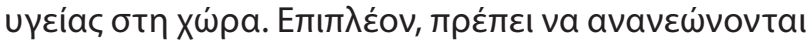

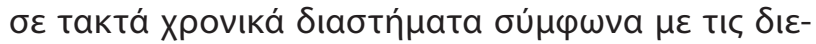

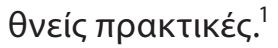

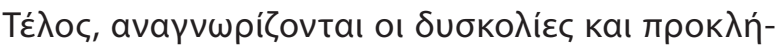

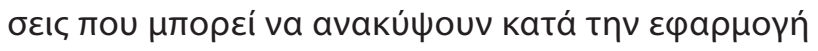

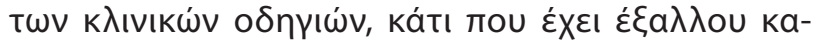

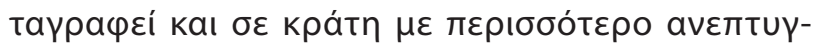

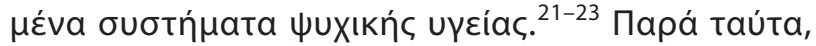

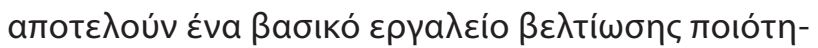

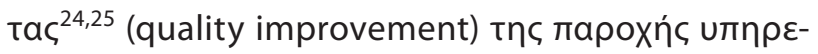

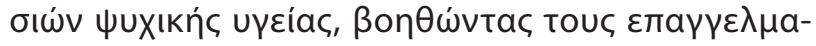

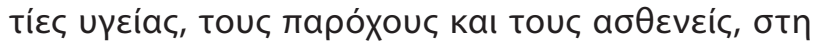

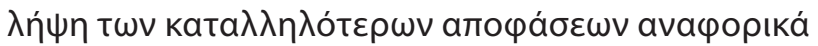

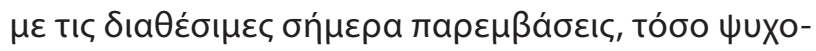

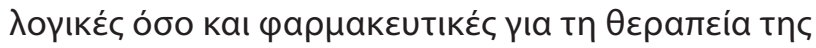

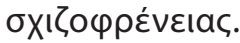

\title{
Clinical guidelines for the management of schizophrenia: Pharmacological and psychological interventions (III)
}

\author{
M. Hadjulis, ${ }^{1}$ M. Margariti, ${ }^{2}$ M. Lazaridou, ${ }^{3}$ G.F. Aggelidis, ${ }^{4}$ \\ V. Fotopoulos, ${ }^{5}$ L. Markaki, ${ }^{6}$ F. Koulouri ${ }^{7}$ \\ 'Department of Psychiatry, Agioi Anargyroi Hospital, Faculty of Nursing, National and Kapodistrian University of Athens, \\ ${ }^{2} 1$ st Department of Psychiatry, Medical School, National and Kapodistrian University of Athens, \\ ${ }^{3}$ Society of Social Psychiatry \& Mental Health, ${ }^{4}$ Department of Psychiatry, General Hospital of Katerini, \\ ${ }^{5}$ Dromokaiteio Mental Hospital, ${ }^{6}$ Executive Council, Hellenic Psychiatric Association, \\ ${ }^{7}$ Directorate of Mental Health, Ministry of Health, Athens, Greece
}

Psychiatriki 2018, 29:303-315

In 2014 a Working Group was set up by the Greek Ministry of Health, with the objective of formulating Clinical Guidelines for the management of schizophrenia which include recommendations for the pharmacological and the psychosocial treatment of schizophrenia. This Working Group utilized the NICE Guideline (National Institute of Clinical Excellence 2014) for the management of Psychosis \& Schizophrenia as the main guide in the development of the Greek guidelines, and in addition 
the American Psychiatric Association (APA) Practice Guideline for the Treatment of Patients with Schizophrenia (APA, 2004), the Royal Australian and New Zealand College of Psychiatrists clinical practice guidelines for the treatment of schizophrenia and related disorders (Royal Australian and New Zealand College of Psychiatrists 2005), as well as other relevant sources. Furthermore, the Hellenic Psychiatric Association (HPA) established an Expert Committee that contributed with its observations to this exercise. With regard to the pharmacological management of schizophrenia, recommendations for initiating and monitoring drug therapy, treating the first psychotic episode as well as treating the acute as well as the maintenance phase of the illness are included. Also included is the management of treatment resistance as well the management of dual diagnosis patients and rapid tranquilization. The guidelines for the psychological treatment of schizophrenia include general recommendations covering all different types of interventions but also specific suggestions for the psychological interventions that should be utilized in the treatment of schizophrenia. In this article the summary recommendations (incorporating the Hellenic Psychiatric Association comments) regarding the pharmacological, psychological and psychosocial interventions in the treatment of schizophrenia are presented.

Key words: Guidelines, clinical guidelines, schizophrenia, pharamacological treatment, psychological treatment.

\section{Bıßהtoypacpía}

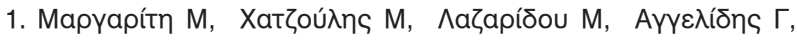

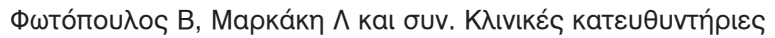

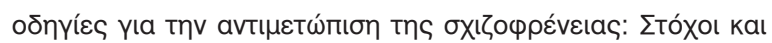

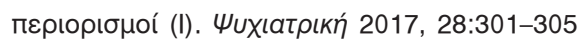

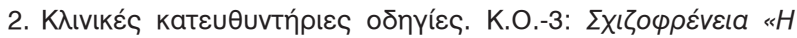

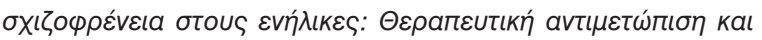

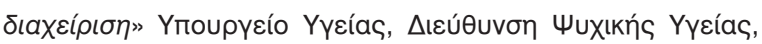
A日ńva, 2015

3. NICE. The NICE Guideline on Core Interventions in the Treatment and Management of Schizophrenia in Adults in Primary and Secondary Care. NICE Clinical Guideline no. 82. London: National Institute of Health and Clinical Excellence, 2009

4. NICE. Psychosis and schizophrenia in adults: treatment and management. NICE Clinical Guideline no. 178. London: National Institute of Health and Clinical Excellence, 2014

5. American Psychiatric Association. Practice Guideline for the Treatment of Patients with Schizophrenia. Washington, DC: American Psychiatric Association., 2004 http://psychiatryonline.org/pb/assets/raw/sitewide/practice_guidelines/guidelines/schizophrenia.pdf (про́бßaơ 6.6. 2014)

6. Lehman AF, Steinwachs DM \& the co-investigators of the PORT Project. Translating research into practice: the Schizophrenia Patient Outcomes Research Team (PORT) treatment recommendations. Schizophr Bull 1998a, 24:1-10, PMID: 9502542

7. Kreyenbuhl J, Buchanan RW, Dickerson FB, Dixon L B. The Schizophrenia Patient Outcomes Research Team (PORT): Updated Treatment Recommendations 2009. Schizophr Bull 2010, 36:94-103, doi: 10.1093/schbul/sbp130

8. Hasan A, Falkai P, Wobrock T, Lieberman J, Glenthoj B, Gattaz WF et al \& World Federation of Societies of Biological Psychiatry (WFSBP) Task Force on Treatment Guidelines for
Schizophrenia. World Federation of Societies of Biological Psychiatry (WFSBP) Guidelines for Biological Treatment of Schizophrenia, part 1: update 2012 on the acute treatment of schizophrenia and the management of treatment resistance. World J Biol Psychiatry 2012, 13:318-378, doi: 10.3109/ 15622975.2012.696143

9. Hasan A, Falkai P, Wobrock T, Lieberman J, Glenthoj B, Gattaz WF et al WFSBP Task force on Treatment Guidelines for Schizophrenia. The World Federation of Societies of Biological Psychiatry (WFSBP) guidelines for biological treatment of schizophrenia, part 2: update 2012 on the long-term treatment of schizophrenia and management of antipsychoticinduced side effects. World J Biol Psychiatry 2013, 14:2-44, doi: $10.3109 / 15622975.2012 .739708$

10. NICE. Violence: The short-term management of disturbed/violent behaviour in in-patient psychiatric settings and emergency departments. NICE Clinical Guideline no. 25. National Institute of Health and Clinical Excellence, London, 2005

11. NICE. Psychosis with coexisting substance misuse. NICE Clinical Guideline no. 120. National Institute of Health and Clinical Excellence, London, 2011

12. NICE. Guidance on the use of electroconvulsive therapy. Technology appraisal guidance [TA59]. National Institute of Health and Clinical Excellence, London, 2003 (updated 2009)

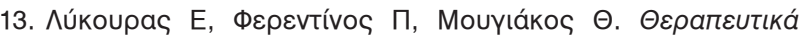

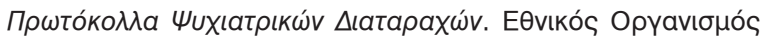

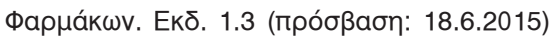

14. Taylor D, Paton C, Kapur S (eds) The Maudsley Prescribing Guidelines in Psychiatry. 11th ed. Wiley-Blackwell, London, UK, 2012

15. Lingford-Hughes AR, Welch S, Peters L, Nutt DJ. BAP updated guidelines: evidence-based guidelines for the pharmacological management of substance abuse, harmful use, addiction and 
comorbidity: recommendations from BAP. J Psychopharmacology 2012 26:899-952, doi: 10.1177/0269881112444324

16. Wobrock T, Soyka M. Pharmacotherapy of schizophrenia with comorbid substance use disorder - Reviewing the evidence and clinical recommendations. Progr Neuropsychopharmacol 2008, 32:1375-1385, doi: 10.1016/j.pnpbp.2008.02.008

17. Asenjo Lobos C, Komossa K, Rummel-Kluge C, Hunger $\mathrm{H}$, Schmid F, Schwarz $\mathrm{S}$ et al. Clozapine versus other atypical antipsychotics for schizophrenia. Cochrane Database Syst Rev. 2010 Nov 10

18. Meltzer H, Alphs L, Green Al, Altamura AC, Anand P, Bertoldi A et al. Clozapine treatment for suicidality in schizophrenia: International Suicide Prevention Trial (InterSePT). Arch Gen Psychiatry 2003, 60:82-91, PMID: 12511175

19. Kane JM, Kishimoto T, Correll CU. Non-adherence to medication in patients with psychotic disorders: epidemiology, contributing factors and management strategies. World Psychiatry 2013, 12:216-226, doi: 10.1002/wps.20060

20. Koola MM, Wehring HJ, Kelly DL. The Potential Role of Longacting Injectable Antipsychotics in People with Schizophrenia and Comorbid Substance Use. J Dual Diagn 2012, 8:50-61, PMID: 22754405

21. Haddock G, Eisner E, Boone C, Davies G, Coogan C, Barrowclough $C$. An investigation of the implementation of NICErecommended CBT interventions for people with schizophrenia. J Ment Health 2014, 23:162-165, doi: 10.3109/ 09638237.2013 .869571
22. Barbui C, Girlanda F, Ay E, Cipriani A, Becker T, Koesters M. Implementation of treatment guidelines for specialist mental health care. Cochrane Database Syst Rev. 2014 Jan 17;(1): CD009780

23. Forsner T, Hansson J, Brommels M, Wistedt AA, Forsell Y. Implementing clinical guidelines in psychiatry: a qualitative study of perceived facilitators and barriers. BMC Psychiatry. 2010, 10:8, doi: 10.1186/1471-244X-10-8

24. Grol R, Grimshaw J. From best evidence to best practice: effective implementation of change in patients' care. Lancet 2003, 362:1225-1230, doi: 10.1016/S0140-6736(03)14546-1

25. Wobrock T, Weinmann S, Falkai P, Gaebel W. Quality assurance in psychiatry: quality indicators and guideline implementation. Eur Arch Psychiatry Clin Neurosci 2009, 259(Suppl 2):219-226, doi: 10.1007/s00406-009-0072-7

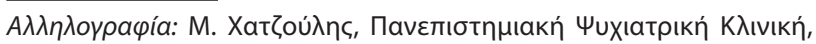

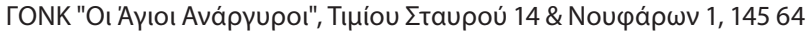

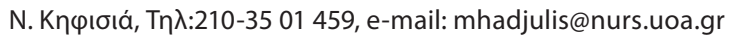

